\title{
Single-session and training effects of focused-attention meditation on neurophysiological and behavioural correlates of cognitive control during motor sequence learning
}

\author{
Russell W. Chan ${ }^{1,2}$, Phillip M. Alday ${ }^{4}$, Lena Zou ${ }^{1,3}$, Kurt Lushington ${ }^{3}$, \\ Mathias Schlesewsky ${ }^{1,3}$, Ina Bornkessel-Schlesewsky ${ }^{1,3}$ and Maarten A. Immink ${ }^{1,2}$
}

${ }^{1}$ Centre for Cognitive and Systems Neuroscience, University of South Australia, Adelaide, South Australia

${ }^{2}$ School of Health Sciences, University of South Australia, Adelaide, South Australia

${ }^{3}$ School of Psychology, Social Work and Social, University of South Australia, Adelaide, South Australia

${ }^{4}$ Max Planck Institute for Psycholinguistics, Nijmegen, The Netherlands

*Corresponding author:

Russell W. Chan

Email: russellchanweili@gmail.com

This research was supported by the Australian Government Research Training Program Scholarship for Russell W. Chan 
Recent theoretical models outline that motor sequence learning involves cognitive control processes that affect stimulus- or plan-based control, and yet clear contributions from cognitive control have not been delineated. Previously, we found that single-session focused attention meditation (FAM) enhanced sequence learning through stimulus-based control which alludes to increased top-down activation. In the present experiment, we were interested in understanding the impact of single-session compared to short-term training on motor sequence learning. Moreover, we proposed to examine the impact on cortical processes more directly using event-related potentials (ERP). Specifically, we investigated the N200 component that is closely related to top-down activation, and the error-related negatively (ERN) component that is closely related to error processing for plan development. 29 participants were randomised to one of three conditions reflecting the level of FAM experienced prior to a serial reaction time task (SRTT): 21 sessions of FAM (FAM21, N=12), a single FAM session (FAM1, N=9) or no preceding FAM control (Control, $\mathrm{N}=8$ ). Continuous 64-channel EEG were recorded during SRTT whereby N200 amplitudes for correct trials, and ERN using mean difference in amplitudes for correct and error trials, were extracted. Component amplitudes, topography and behavioural outcomes were compared using linear mixed effects regression models between groups. Firstly, FAM21 exhibited faster reaction time performances in majority of the learning blocks compared to FAM1 and Control. FAM21 also demonstrated a significantly more pronounced N200 component over all anterior and the central regions during SRTT compared to FAM1 and Control. When N200 amplitudes were modelled against general learning performance, FAM21 also showed the greatest rate of decline over all anterior and the central regions during SRTT compared to FAM1 and Control. However despite our expectations, no robust group differences over the central-midline regions for the ERN were observed. Turning back to our block-on-block RT performances results support that top-down activation was further increased after short-term FAM training, to support better RT performance compared to the other groups. Importantly, short-term FAM training facilitates more dynamic modulation of top-down activation: greater levels of activation supported early improvements of general practice effects, whilst later improvements required significantly less top-down compared to the other groups. 
Sequenced actions feature prominently in everyday life, although less clearly understood are the underlying cognitive control processes that determine the manner that they are acquired. Sequential action is ultimately the result of the outflow arising from cognitive control that are shared by other behaviours including those that do not involve movement. Recent theoretical works have highlighted the importance of these cognitive control processes and that the level of top-down control will affect sequence learning control during acquisition (Tubau, Hommel, et al., 2007; Verwey et al., 2015). To bridge understanding in the cognitive control of motor sequence learning, we have shown that mental training in the form of single-session focused attention meditation (FAM) can affect states of top-down control during learning. Specifically, FAM was shown to strengthen top-down activation and in turn improve the performance of subsequent sequence learning (Chan et al., 2017; Chan, Immink, \& Lushington, In review; Immink et al., 2017). In this experiment, we aimed to further understand the cognitive control of sequence learning by investigating the neurophysiological correlates of sequence learning that has thus far been lacking. We are also guided by the current evidence from the neurophysiological effects of FAM that modulate top-down activation of cognitive control (Cahn \& Polich, 2006; Larson et al., 2013; Malinowski, 2013; Moore et al., 2012), to form our predictions of how sequence learning control will be affected. We provide a brief review of evidence for cognitive control in sequence learning, FAM effects on cognitive control and in turn effects on sequence learning control, and the key neurophysiological correlates that will index the cognitive control of sequence learning.

\section{Cognitive control in motor sequence learning}

In one of the most concise definitions, Abrahamse and colleagues (2013) outlines what is considered the epitome of motor sequence learning: the acquisition of the skill to rapidly and accurately produce a sequence of movements with limited effort and/or attentional monitoring. Deceivingly simple in definition, although the exact contributions and relationships in the core elements such as speed, accuracy, effort and attention have yet to be clearly delineated. This however has led to an increasing amount of motor sequence learning research that draws upon the contributions of cognitive control to explain how the human system represents and acquires sequences differently (Abrahamse et al., 2010; Tubau, Hommel, et al., 2007; Verwey et al., 2015). For example, Verwey et al. (2015) outline that perceptual information from stimuli during sequence learning are processed by a central 
91 processor before motor processes execute the sequence of movements usually aimed towards

92 efficiency. This central processor is thought to have significant contributions from cognitive control (Verwey et al., 2015) by organising sequence representations that orientate either toward stimuli or responses (Abrahamse et al., 2010).

Although other motor sequence learning paradigms such as the discrete sequence production task (Abrahamse et al., 2013) have been designed to investigate cognitive contributions, this experiment focuses on the Serial Reaction Time Task (SRTT: Nissen \& Bullemer, 1987). In the SRTT paradigm, different forms of sequence learning can be differentiated. For example, the difference in mean reaction time (RT) between the last learning block relative to the first learning block discerns general learning performance. General learning performance is closely linked to what is known as a reinforcement of stimulus-to-response (S-R) mapping (Abrahamse et al., 2010), whereby the stimulus is used as main source of input information and signals for a specific response that is not elaborated in the context of a sequence. Tubau et al. (2007) also describe this as stimulus-based control, where performance improvement is evident even when the task is lacking a sequence which is why they are considered general practice effects (Abrahamse \& Noordzij, 2011).

Another way to understand sequence learning in the SRTT is to compute the difference in MRT between the last learning block relative to the transfer block, and this is known as sequence-specific learning. The premise for this measure is that if participants have acquired a large portion of the sequence, then by providing a transfer task containing random stimuli will disrupt responses greatly. The learner would be fixated on providing responses that have been mapped by the original sequence (Robertson, 2007). Greater increases in RT indicate higher levels of sequence-specific learning. Sequence-specific learning is closely related to what Abrahamse at al. (2013) refer to as response-to-response (R-R) mapping, where responses are treated as the main source of input information and successively linked to each other. Tubau et al. (2007) refers to this as plan-based control because as responses are linked, an internal representation of the sequence is being built upon which develops into an action plan for accurate motor responses (Hommel, 1996; Tubau, Hommel, et al., 2007). The efficiencies developed are specific to an internalised action plan of the practiced sequence, which further explains why performance gains are lost when there is deviance from the learnt sequence structure. A major contributor that influences motor sequence learning representations are cognitive control operations at the level of information processing (Abrahamse et al., 2010; Hommel, 1996; Hommel, 2015; Hommel et al., 2001; Tubau, Hommel, et al., 2007). 
Cognitive control is defined as set of higher-order cognitive operations that performs planning, decision making, maintaining relevant and/or inhibit irrelevant information, in order to perform goal-directed performances successfully (Amer et al., 2016; Gratton et al., 2017; Szczepanski \& Knight). In sequence learning, cognitive control actively manages the interplay between perceptual, central and motor representations (Verwey et al., 2015; Verwey \& Wright, 2014) and a suggested unitary link that manages this interplay is the attentional function and its control (Miyake et al., 2000). Essentially, attentional function has been described to operate like a "zoom lens", in which the aperture has an ability to adopt a narrow (tunnelled), mid or broad (attending to many global features) range in a continuum based on the engaged task (Lutz et al., 2015). Curran \& Keele (1993) provided one of the earliest discoveries to show that motor sequence learning is sensitive to attentional manipulations in that attentional learning facilitates the encoding of events within the context of the sequence. In contrast, nonattentional learning operates simple associations for responding. This is similar to what was described earlier as stimulus- and plan-based control, which indicates that these control modes can be biased by attention either directed towards stimuli or responses during learning. Cognitive control has also been found to be contextually driven and doubleedged, whereby varying levels of activation can benefit some tasks and yet at the same time detrimental for others (Amer et al., 2016; Amer \& Hasher, 2014). For example, increased top-down control appears to be of benefit for tasks that require focused attention and to suppress distraction, while increasing the speed of processing (Lustig et al., 2006). On the other hand, a weakened top-down shows benefit when tasks require more diffused attention to incorporate a variety of stimuli for creative solving, and learning of statistical regularities (Schwab et al., 2016). If we consider that the level of top-down activation is what determines the control of attention, we can extrapolate this towards motor sequence learning where states of increased top-down control may bias stimulus-based control, and that states of weakened top-down control may bias plan-based control. amongst other goal-directed activities including those that do not involve movement. Recently investigations from one such goal-directed activity in the form of focused-attention meditation (FAM) has gained increasing evidence for its ability to modulate cognitive control operations in positive ways such as improved attentional control (Jha, Krompinger, \& Baime, 
attention on an object such as the breath or awareness of body parts (Lutz et al., 2015; Slagter et al., 2011). FAM trains attentional control by performing the cognitive control operations required to maintain relevant and/or inhibit irrelevant information in a goal-directed manner (Gallant, 2016; Miyake et al., 2000). Specifically, FAM aims to sustain attention on the object, to monitor and recognise distraction when it occurs, to disengage from the distraction and shift attentional focus back to the original object (Gallant, 2016; Malinowski, 2013; Tang et al., 2015). It is thought that attentional control improves due to the practice of these processes that occur in a cyclical manner (Hasenkamp \& Barsalou, 2012; Malinowski, 2013).

Recent evidence have found that as little as one single-session of FAM in naïve participants, was able to bias cognitive control and induce states of increased top-down control that remained inert in subsequent cognitive tasks (Colzato, Sellaro, Samara, Baas, et al., 2015; Colzato et al., 2017; Colzato et al., 2016). Specifically, Colzato, Sellaro, Samara, Baas, et al. (2015) found that FAM practice prior to performing a rapid serial visual presentation task (RSVP) resulted in a larger attentional blink when the lag time was extremely short between target and presentations. It was interpreted that FAM practice reinforced top-down control, which resulted in a narrow and increased attentional focus on the initial target but not both targets. Colzato et al. (2016), also found that FAM practice lead to faster performances for incongruent trials during a global-local task performance. This was also consistent with the notion that FAM practice resulted in a state of strengthened top-down control which suppressed irrelevant information and prioritised local competition processing. In direct applications of FAM for motor sequence learning, when a single-session of FAM immediately preceded learning, it was shown that stimulus-based control was responsible for reduction in response times and improvement in general learning performance, which further supports that FAM induces states of strengthened top down (Chan et al., 2017, In review). However, when FAM was temporally more distant relative to motor sequence learning, effects were different, and showed that plan-based control supported greater levels of improvements in sequence-specific learning, attributed to states of weakened top-down whilst learning. Similarly, Immink et al. (2017) also found that a single-session FAM practice also facilitated greater levels of general learning performance but not sequencespecific learning when modulated by low levels of cognitive effort engagement during FAM practice. The current evidence is consistent in support that single-session FAM practice can induce states of strengthened top-down cognitive control that remain inert during subsequent cognitive tasks and motor sequence learning. The effects also appear to suggest that this strengthened top-down, bias stimulus-based control for increased general learning effects 
during motor sequence learning. It would therefore be important to form research to understand if single-session FAM effects are enhanced by a bout of short-term FAM training, and if so, the manner by which effects influence sequence learning. It is conceivable to expect that training may strengthen top-down control effects, but such predictions should be tested to establish clearer understanding. It should also be highlighted that the evidence presented thus far has been predicated mainly on behavioural measures to infer cognitive control effects that occur in the cortex. This is a limitation because behavioural measures are a net outcome of different cognitive processes, making it difficult to understand their relative contributions (Müller \& Knight, 2002), and prevents conclusive inferences on mechanistic actions by which FAM exerts cognitive control effects. To overcome the issue of reliance on behavioural data, acquisition of more direct neurophysiological correlates such as eventrelated potentials (ERP) maybe be able to strengthen these inferences.

\section{N200 and error-related negativity in motor sequence learning and meditation}

The key advantage for the use of ERP in this experiment, is that the methodology provides a much more precise estimation as to changes in neuronal processes involved in cognitive control operations (Luck et al., 2000; Müller \& Knight, 2002). The N200 component is particularly relevant because of sensitivity to attention and cognitive control for performance monitoring (Ullsperger, Fischer, et al., 2014). Because of its timing, the N200 has been found to be representative of attentional processes such as orienting (Folstein \& Van Petten, 2008), stimulus discrimination (Ritter et al., 1983; Satterfield et al., 1990) and target selection (Donchin et al., 1978; McCallum, 1980). During performance monitoring, the N200 is generated when attending stimuli and is affected by congruency (Näätänen, 1990). Voltage differences in the component between congruent and non-congruent trials provide insight to the cognitive processing differences towards stimuli (Patel \& Azzam, 2005). In addition, the anteriorly-distributed N200 component was also found to be sensitive to cognitive control (Folstein \& Van Petten, 2008) and that fluctuations in voltage maybe an indexing the level of top-down control regulation (Jack et al., 1980). Higher voltages may therefore be an indication of strengthened top-down control for processing information related to attentional processes, while lower voltages may be an indication of weakened top-down.

Although ERP recordings have been used to understand the N200 component during SRTT sequence learning performance, majority of the studies commonly aimed to understand contributions from the awareness of sequence in participants and not from approach of cognitive control (Baldwin \& Kutas, 1997; Eimer et al., 1996; Ferdinand et al., 2010; 
Russeler et al., 2003; Russeler \& Rosler, 2000). A consolidated but limited conclusion from the study of Russeler \& Rosler (2000) is that increased N200 amplitudes were found for learners aware of the sequence for both perceptual and motor deviants, compared to learners who were not aware of the sequence. If we extrapolate this finding and reinterpret it from a cognitive control perspective, increased N200 amplitude for deviants is indicative of sequence disruption the current representation (Daltrozzo \& Conway, 2014). An increased top-down activation (greater amplitude) for cognitive control is firstly required to resolve the deviance and to maintain equal or greater levels of performance (Danielmeier et al., 2011; Folstein \& Van Petten, 2008). It also implies that top-down control related changes in the N200 amplitude (regardless of congruency) may serve as a signal that detects fluctuations in cognitive control for the maintenance of performance. In this case, gradients of change in amplitude will therefore be representative of top-down control to resolve a particular stimulus at any given point in time (Alexander \& Brown, 2010; Daltrozzo \& Conway, 2014; Folstein \& Van Petten, 2008; Wickens et al., 2013). Because top-down control is linked to stimulusbased control in motor sequence learning (Tubau, Hommel, et al., 2007; Verwey et al., 2015), the N200 may therefore be direct representation of top-down activation for stimulus-based control. Increased top-down would result in greater N200 amplitudes; and that changes over time during sequence learning, are representative of learning-related top-down control changes (Daltrozzo \& Conway, 2014).

To investigate the changes of the N200 from FAM training, Moore et al. (2012) studied the effects of 10-minute daily FAM training over a 16-week for ERP changes associated with the attentional processes in the Stroop task. It was found that meditation training led to a relative increase in N200 amplitudes in both hemispheres regardless of congruency, even though the authors failed to detect behavioural differences in Stroop performance. It was surprising that the authors did not attempt to correlate the behavioural results to the N200 amplitudes, to establish further differences between the meditators and controls. In another study by van Leeuwen, Singer, \& Melloni (2012), it was found that meditators proficient in FAM had a more constrained attentional control and performed faster in local target levels in global-to-local paradigm (indicative of increased top-down) than meditators proficient in another style. In addition, meditators were also found to have greater negativities compared to controls in the N200 during the global-to-local task processing. The current evidence appears to suggest that FAM training will strengthen states of increased topdown control which is reflected by greater N200 amplitudes during cognitive task performance compared to other control conditions. Currently, there are no studies that have 
investigated the effects of single-session meditation on N200 amplitude changes during cognitive task performances or motor sequence learning.

\section{Error-related negativity in motor sequence learning and meditation}

Another index of cognitive control in sequence learning recently being investigated is the error-related negativity (ERN) (Beaulieu et al., 2014; Krigolson et al., 2009). The ERN, is a negative-going response-locked ERP component that occurs approximately within the first $150 \mathrm{~ms}$. As the ERN is usually distributed over the anterior-midline areas of the scalp, it is suggestive of contributions from the anterior cingulate cortex as neural generators for the component (Botvinick et al., 2001; Ullsperger, Fischer, et al., 2014). During sequence learning, cognitive control pathways monitor and detect erroneous responses and allocate cognitive resources for the implementation of adaptive strategies (Botvinick et al., 2001; Carter et al., 1998). The mismatch hypothesis (Gehring et al., 1993) is an interpretation that supports the role of cognitive control in sequence learning. Specifically, it considers that a comparator system in cognitive control continuously evaluates the similarities between the neural representations of the initiated response and the correct response (Holroyd \& Coles, 2002). When a mismatch between the representation and response occurs, the ERN is produced to signal for adaptation for future responses to reinforce future learning (RodriguezFornells, Kurzbuch, \& Munte, 2002).

Recently, Beaulieu et al. (2014) designed a study to establish the relationship between sequence learning in the SRTT, cognitive control and the ERN. In their study, participants performed a standard SRTT while ERPs were recorded. The authors aimed to compute several analyses to establish relationships between early learning (first 4 blocks) to late learning (last 4 blocks) ERN amplitudes against general and sequence-specific learning performance. Their first finding was the mean ERN amplitude across all learning blocks correlated inversely with mean response accuracy (higher accuracy and more negative ERN) supporting the ERN serves to enhance modulation for future performance. Their second finding was that mean ERN change amplitude (between early and late learning) significantly correlated with general learning improvements $(r=.487)$. However, mean ERN change amplitude had an even better correlation with sequence-specific learning than general learning improvements $(r=.515)$. In another recent study investigating ERN and sequence learning, further provides evidence of how the ERN is modulated against sequenced and random stimuli (Rüsseler, Münte, \& Wiswede, 2018). It was found that errors made for sequenced stimuli had greater ERN negativities compared to random stimuli, to which the authors 
attributed that the cognitive system was able discriminate that errors during sequence stimuli was used for updating the working memory model of the sequence. In contrast, reduced ERN negativities in random trials indicates less relevancy for the working memory model. The most interesting finding was that over the course of learning, ERN amplitudes for errors in structured stimuli became increasingly more negative indicating that errors are more significantly discriminated as violations to an established internalised plan of the sequence. These results were in agreement with Beaulieu et al. (2014) that sequence-specific learning is associated with the notion of strengthening R-R mappings and the development of an internalised plans. It would point to the role that the ERN maybe indexing cognitive control related more closely to plan-based control, although it may also be highly related to general learning as highlighted earlier (Beaulieu et al., 2014).

No studies have investigated ERN and meditation in the context of sequence learning. In addition, the evidence for the effects of meditation and ERN in other cognitive task are also less clear. In a cross-sectional study between meditators with one-year of experience and non-meditators, Teper \& Inzlicht (2013) found that meditators had enhanced ERN amplitudes following error commission during the Stroop task compared to non-mediators, although no differences in behavioural error performances between the groups were found. The authors attributed the differences to indirect effects of conflict regulations skills developed in meditation, which muddles the conclusions. In addition, cross-sectional studies greatly reduce the power to infer direct contributions from meditation practice. In a single-session FAM study design, Larson et al. (2013) studied the ERN elicited during errors in a Flanker Task but found no differences in the ERN between naïve participants that performed FAM prior to testing and controls. The behavioural results in the Flanker task also did not reveal any group differences in accuracy, error rates or post-error slowing. This suggests that singlesession FAM may not exert a large influence on the cognitive control processes related to the ERN. It has been suggested that a period of longer training might provide greater effects in ERN and error processing, this has yet to be tested (Larson et al., 2013; Tang et al., 2015).

\section{The present experiment}

In our previous work, we showed that single-session FAM induced cognitive control states of increased top-down that subsequently promoted stimulus-based control during subsequent motor sequence learning (Chan et al., 2017, In review; Immink et al., 2017). In the present experiment, we aimed to replicate these behavioural findings but also to further improve inferences on top-down activation by recording ERPs that are more direct representations of 
cognitive control. Current evidences suggests that the N200 is representative of top-down control (Folstein \& Van Petten, 2008; Russeler \& Rosler, 2000) and might be closely related to stimulus-based control and general learning performance (Abrahamse \& Noordzij, 2011). In contrast, the ERN has been shown to be representative of cognitive control activation that is related with prediction errors (Gehring et al., 1993; Holroyd \& Coles, 2002) internal plan development (Richardson et al., 2013), and sequence-specific learning (Beaulieu et al., 2014; Rüsseler et al., 2018), and therefore a more direct representation for plan-based control (Tubau, Hommel, et al., 2007). As an improvement over correlational methods, we aimed to use a model-based approach to test and establish links between the N200 and stimulus-based control, and between the ERN and plan-based control.

In the present experiment, we aimed to replicate the effects of a single-session of FAM and predict that biased states of enhanced top-down cognitive control in naïve participants will be resultant during subsequent sequence learning. Specifically, increased N200 negativities will be reflective of enhanced top-down effects and performance will rely primarily on stimulus-based control under the notion of general practice effects. We also wanted to understand the effects of short-term FAM training (20 sessions/ once daily) and predict that states of strengthened top-down cognitive control will be even greater than singlesession FAM practice. Therefore, we predict that N200 amplitude during sequence learning will be further increased after a single-session of FAM that follows brief FAM training. Increased N200 amplitude would be reflective of further reinforcement of top-down control effects and thus promotion of stimulus-based control and general practice effects.

However, current evidence also suggests that single-session FAM (Saunders, Rodrigo, \& Inzlicht, 2016) and that FAM training (Tang et al., 2015; Teper \& Inzlicht, 2013) may result in enhanced processing of errors and therefore increased ERN. There are no establish guidelines on the length of training but we wanted to understand if short-term FAM training would lead to the enhancement of error-processing compared to single-session effects. Therefore, we predict that increased ERN amplitudes will be reflective of enhanced modulation for error processing and that learning performance would be in line with a reinforcement of plan-based control and sequence-specific learning effects. 


\section{Participants}

356

357

358

359

360

361

362

363

364

365

366

367

368

369

370

371

372

373

374

375

376

377

378

Thirty-three volunteers responded to recruitment from flyers placed in university campuses around Adelaide, and online advertisements from the Facebook platform that targeted $18-35$ year old adults living within $25 \mathrm{~km}$ radius of Adelaide city in Australia. Our exclusion criteria were: participants must not practice any form of meditation regularly in the last year; or taken part in any motor learning study in the last year (checked via our experimental database); must be naturally right-handed with normal or corrected vision; possess English skills equivalent to the level of a native English speaker; be physically healthy without any learning disabilities; free from mental health issues and neurological disorders. Typists, piano players, gamers or professionals that engage in high levels of fine finger movements were excluded. Lastly, they must agree and be willing to perform a mental training task daily that last $22 \mathrm{~min}$ and report on their practice via an online form.

Of the 33 participants, one participant ceased participation at the second data collection session. Following completion of data collection, three more participants were excluded; one participant revealed to be a regular meditation practitioner in the past; one participant did not practice the mental training correctly; one participant had incorrectly acquired EEG recordings. Data from 29 participants (18 females, $23.1 \pm 4.4$ years; $100 \%$ self-reported right-handed) remained for final analysis. Participants were unaware of the experiment aims during recruitment and provision of experiment-related information. The term 'meditation' was not used during the experiment to minimise participation bias. The research protocol was approved by the University of South Australia Human Research Ethics Committee and all participants provided written informed consent prior to participation. If participants completed all four data collection sessions, they received AU\$100 to compensate for their time and travel cost associated with participation in the experiment. 
The SRTT (Nissen \& Bullemer, 1987) has been used in conjunction with EEG to understand the neurophysiological correlates of cognitive control in sequence learning (Beaulieu et al., 2014). The stimuli in the current experiment's SRTT involved a row of four hollow boxes that were spatially mapped to four response keys on a modified keyboard that were numbered 1 to 4 from left to right. The participants used their index and middle fingers of the left (middle finger on key 1 and index finger on key 2) and right hand (index finger on key 3 and middle finger of key 4) spatially mapped to the numbered keys for responding. The response key was signalled when one of the four boxes was filled in red.

Stimuli was presented using E-Prime ${ }^{\circledR} 2.0$ Software (Psychology Software Tools Inc., Sharpsburg, USA) on a 21-inch Velta DE-770 BA (Dongguan, China) CRT monitor running at $1680 \times 1050$ pixel resolution in 64-bit colour with a screen refresh setting of $100 \mathrm{~Hz}$. At a sitting distance of $60 \mathrm{~cm}$ from the display (not strictly enforced), the visual angle dimension of each box was $2^{\circ} \times 2^{\circ}$ with a $2^{\circ}$ separating each box. After each response, a $500 \mathrm{~ms}$ response-stimulus interval (RSI) occurred before the presentation of the stimulus for the next trial. The duration of the RSI was set to ensure that previous trial influences did not affect the ERP recordings between trials. Inaccurate responses were notified with an error message ("Key Error") and all four boxes were marked with an "X" in the centre. At the end of each block, performance feedback was provided in terms of block averaged reaction time (RT) and count of error trials. Following feedback presentation, participants received a $30 \mathrm{~s}$ rest interval. ERP event markers were recorded at the start of each block (indicating block number), at trial presentation (stimulus), at response initiation (response) and a separate marker for whether the response was a correct or erroneous. These event marker provided segmentation of epochs for ERP analysis described in a later section. Please see Figure 1.1 (Chapter 1) for a pictorial representation of the SRTT stimuli.

In the Familiarisation session (baseline), participants engaged in the SRTT that was organised into three familiarisation blocks $1-3$. Each familiarisation block consisted of 120 pseudorandom stimulus presentation schedules with the condition that stimulus presentation was not repeated on consecutive trials and presented with equal proportion in each block. A trial represented the presentation of one stimulus and the production of one response. In the final Learning session (post-training), participants engaged in the SRTT that was organised into 12 sequence learning blocks. Each sequence learning block comprised of 120 trials per block and a 12-item second order conditional (SOC) sequence (i.e. 121342314324; Reed \& 
Johnson, 1994). Following completion of sequence learning blocks, participants then completed one transfer block (Block 13) involving only pseudorandom trials presented in the same manner as the familiarisation blocks, and a final sequence learning block (Block 14), where the learnt SOC sequence was re-introduced.

\section{Focused-attention meditation (FAM) and Control Task}

Guided by the meditation taxonomy by Nash and Newberg (2013), the current experiment operationalised yoga nidra as a cognitive-directed type of FAM that involves four stages: preparation, directing attention to body regions, directing attention to natural breathing and completion (Saraswati, 2002). The FAM technique was shown to bias cognitive control in subsequent sequence learning and the full transcript was reported in Chan et al. (2017). The yoga nidra was performed in a seated position and the eyes were closed, their palms turned upwards resting on their thighs for the duration of $22 \mathrm{~m}$. Instructions were audio-recorded using the voice of a male professional meditation instructor.

Rigour in meditation research is important, and studies are encouraged to include active-control conditions that match modality and comparable to meditation practice (Davidson \& Kaszniak, 2015; Hasenkamp et al., 2012). To fulfil this, we included a control task in the form of an audio listening task (LT). The goal was to suitably contrast FAM against another mental listening task for activity and practice equivalence. We operationalised a set of instructions that guided the listener to mentally perform several steps in the maintenance of a garden. The delivery of LT was procedural information but did not instruct directing or control of attention like FAM. This was to understand if the content and cognitive control processes in FAM were unique compared to attentive listening. The LT instructions were compiled from the audiobook “ABC of Vegetable Gardening”, Track 9, Chapter 11, Part A (Rexford. 1916) from the Internet Archive https://archive.org (direct link: https://archive.org/details/abcvegetablegardening_1412_librivox). Track duration was edited to ensure duration equivalence to FAM. Usage of the audio tracks for the purpose of this research was under a Creative Commons license.

In the laboratory, both FAM and LT conditions were played using the E-Prime ${ }^{\circledR} 2.0$ Software environment, using a stereo Altec Lansing (New York, USA) BXR 1220 audio speakers at a fixed volume of $40 \mathrm{db}$. All participants were instructed that the aim in both conditions was to maintain their attention and mentally follow the audio instructions as best as possible, to remain awake and physically still. For their home practice, participants were 
asked to practice as closely as possibly to the same manner performed in the laboratory, although not strictly enforced.

\section{Electrophysiological recordings and pre-processing}

EEG was recorded continuously from $64 \mathrm{Ag} / \mathrm{AgCl}$ electrodes (ActiCAP, Brain Products) with a BrainVision actiCHamp Active Electrodes amplifier system (Brain Products GmbH, Gilching, Germany). For monitoring eye movements and blinks, the horizontal and vertical electrooculogram (EOG) was recorded with supra- and infraorbital electrodes on the left eye and two electrodes placed next to the external canthi of the left and right eyes. EEG and EOG were sampled at $512 \mathrm{~Hz}$ and impedances were kept below $5 \mathrm{k} \Omega$ during recording. Two additional electrodes (left and right mastoids) were used as online reference. Offline preprocessing of data was performed in MNE-Python v0.15 (Gramfort et al., 2013). EEG data was re-referenced to the average of the left and right mastoids to avoid asymmetrical effects. The data was filtered with a digital phase-true finite impulse response (FIR) band-pass filter $(0.3-30 \mathrm{~Hz})$. Epochs containing eye blinks and artefacts were detected and removed via a customised MNE script (see appendix 2) and the EOG rejection criterion was $150 \mu \mathrm{V}$. Individual averaged ERPs were segmented to obtain epochs starting 200ms prior to and $800 \mathrm{~ms}$ following stimulus event marker of the SRTT, and starting $200 \mathrm{~ms}$ prior to and $800 \mathrm{~ms}$ following response event marker of the SRTT

\section{Procedure}

Please see Figure 4.1 for the experimental procedure. During the first Familiarisation session, participants were explained their involvement in the study and provided informed consent. Once consent was given, participants were fitted with the BrainVision EEG actiCAP to record ERPs, and then informed that they were to perform three familiarisation blocks of a keypressing task. Participants received written instructions for the SRTT familiarisation on the computer monitor on the use of appropriate fingers and to press the response keys that corresponded to the stimuli as accurately as possible. Response speed and accuracy was emphasised of equal importance. Once ready, participants completed three SRTT Familiarisation blocks (1-3) involving pseudorandom stimulus presentation, which allowed for participants to familiarise responding to the SRTT. The results in this session were representative of participants' baseline behavioural and ERP performance.

The next day, participants were invited back to the laboratory for their Baseline EEG collection session associated with the cognitive training conditions they were randomly 
476 allocated to. There were three experimental conditions that determined the daily cognitive

477 training they would engage in. Participants allocated to the FAM21 condition were required

478 to practice FAM once-a-day for the next 19 days for their cognitive training (following

479 session one in the lab), and on the final data collection session, perform a single-session of

480 FAM prior to SRTT learning. Those in the FAM1 condition were required to practice the

481 control LT for the next 19 days for their cognitive training, and on the final data collection

482 session, perform a single-session of FAM prior to SRTT learning. In the Control condition,

483 participants were required to practice the LT for the next 19 days as part of their cognitive

484 training and on the final data collection session, perform a single-session of the LT prior to

485 SRTT learning. This Baseline session was considered each participants' first day of cognitive

486 task practice and EEG data was recorded. This allowed participants to ask any questions

487 about the task, and to establish baseline EEG performance during cognitive task engagement.

488 On day 14 of daily cognitive training, participants returned to the laboratory to perform their

489 cognitive practice whilst EEG was recorded.

$490 \quad$ Following completion of 20 days of training in FAM or LT, participants returned to

491 the laboratory for a final session of EEG recording whilst performing single-session FAM or

492 LT practice (Day 21), followed by sequence learning. After completion of the EEG

493 recording, we checked equipment and ensured that EEG impedances were still below $5 \mathrm{k} \Omega$

494 before starting the ERP recordings for SRTT performance. This process took approximately

$49510 \mathrm{~min}$. Once ready, participants were then presented instructions to participate in the SRTT

496 and again reminded that response speed and accuracy were of equal importance for

497 performance. Participants then completed 12 sequence learning blocks $(1-12)$ where

498 unknown to participants, stimulus presentation followed the repeating 12-item SOC sequence.

499 A transfer block (13) was then presented followed by a final block (14) where the original

500 SOC sequence was re-introduced. 


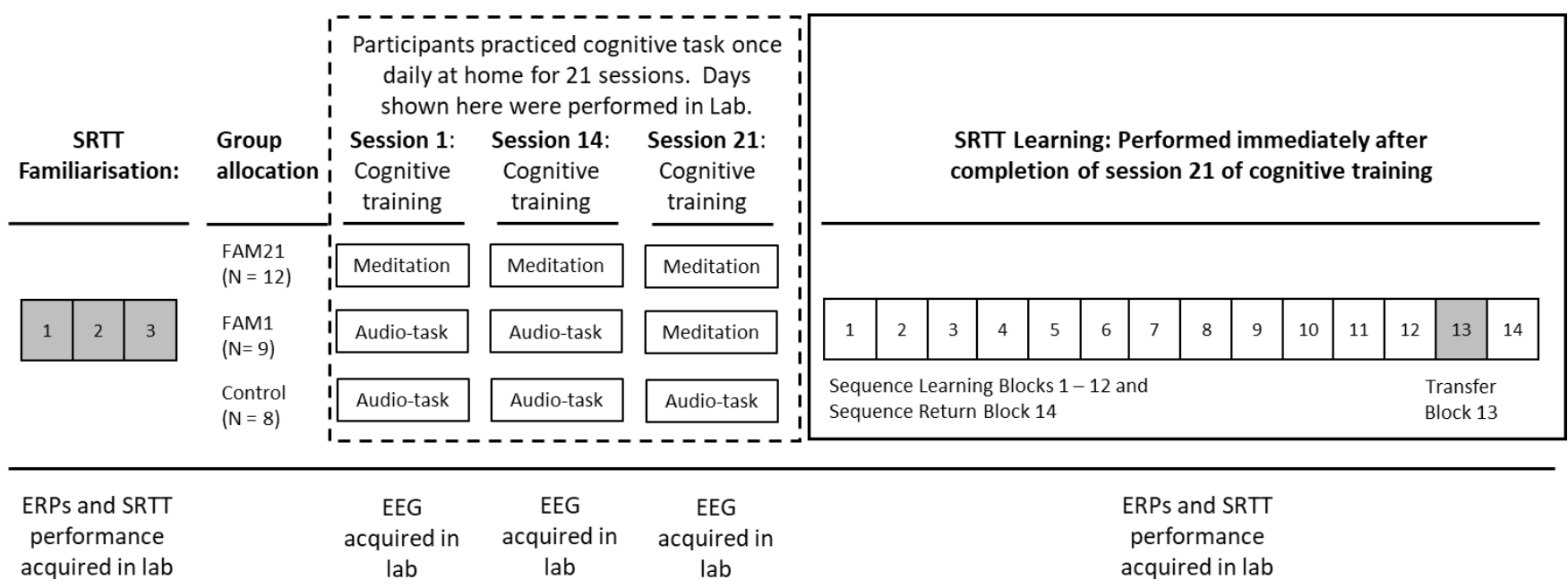

502 Figure 4.1 The experimental procedure. During Serial Reaction Time Task SRTT (SRTT)

503 Familiarisation, participants completed three pseudorandom sequence blocks Participants

504 were then randomly allocated to three different conditional groups that differed on their

505 mental training conditions: FAM21 practiced once daily 22-minute focused-attention

506 meditation (FAM) and after 20 sessions, a single-session of FAM before SRTT; FAM1

507 practiced once daily 22-minute audio listening task as a control condition and after 20

508 sessions, a single-session of FAM before SRTT; Control practiced once daily 22-minute audio

509 listening task and after 20 sessions, a single-session of audio listening task before SRTT.

510 Sessions 1, 14 and 21 were performed in the laboratory whilst EEG data was collected.

511 Immediately after completing session 21 of cognitive training, all participants the performed

512 SRTT. Each SRTT Learning block contained 120 trials and blocks $1-12$ involved a second 513 order conditional (SOC) sequence (121342314324; Reed \& Johnson, 1994). Transfer Block

51413 involved a pseudorandom sequence and, the original SOC sequence presented during

515 Sequence Learning Blocks was reinstated in the final Block 14

\section{Cognitive task manipulation check: Spectral power in the alpha band}

518 The motivation for this manipulation check was based on improving our previous work (Chan 519 et al., 2017), and to increase rigour in meditation (Davidson \& Kaszniak, 2015) and show

520 quantitative differences in mental states between different cognitive tasks. The investigation

521 of power in the alpha band provides an indication for whether a participant is actually

522 spending attention on task or attention spent unrelated to task (i.e. mind-wandering) (Baldwin

523 et al., 2017). Although investigation of EEG frequency band power is relevant, in-depth

524 analysis remains the subject of another publication as the goals do not align well within the 
525 scope of the current experiment. Session 21 of cognitive training, that was conducted in the 526 laboratory was of interest. FAM21 and Control groups performed FAM and LT respectively

527 (final $21^{\text {st }}$ session), whilst the FAM1 group performed a single-session of FAM (first 528 exposure)

529 We segmented the EEG data acquired during the cognitive task performance into a 530 single 22-min epoch in preparation for data processing. All processing was performed on 531 BrainVision Analyzer Ver.2.1. EEG data was re-referenced to the average of the left and 532 right mastoids to avoid asymmetrical effects. The data was filtered with a digital phase-true 533 finite impulse response (FIR) band-pass filter $(0.3-30 \mathrm{~Hz})$. Although the tasks were performed 534 eyes-closed, an Independent Components Analysis (ICA) was performed to identify and 535 remove ocular events. Artefacts were rejected using a criterion set at $10 \mathrm{uV} / \mathrm{ms}$ for voltage 536 step gradients and max-min interval ranges of $150 \mathrm{uV}$. Then, a Fast Fourier Transform (FFT) 537 was performed on channels and participants, which yielded raw spectral power values as $538 \mathrm{uV}^{*} \mathrm{~Hz}$. The alpha bandwidth was 8-13 Hz (Lee, Kulubya, Goldin, Goodarzi, \& Girgis, 2018) 539 and values for each channel for all participants were exported for analysis in R environment 540 Ver. 3.4.3 (RStudio Team, 2015). Electrodes and their averaged amplitudes were then 541 grouped into nine regions of interests (ROI) by laterality and sagittality. Please see the Figure 5424.2 for the layout of electrodes used for region of interest (ROI) analyses for EEG data.

543 Alpha spectral power was analysed with LMER models using the lme4 package (Bates et al., 544 2015) in the R environment. For the model, the outcome variable was - mean spectral power $545\left(\mu \mathrm{V}^{*} \mathrm{~Hz}\right)$ in 22 min cognitive task engagement in Session 21, and the factor variables: (1) 546 Group (i.e. FAM21, FAM1 or Control), (2) Laterality, (3) Sagittality. Subjects was specified as a random factor to control for their associated intraclass correlation (i.e., random intercept models; Oberauer \& Kliegl, 2006). 


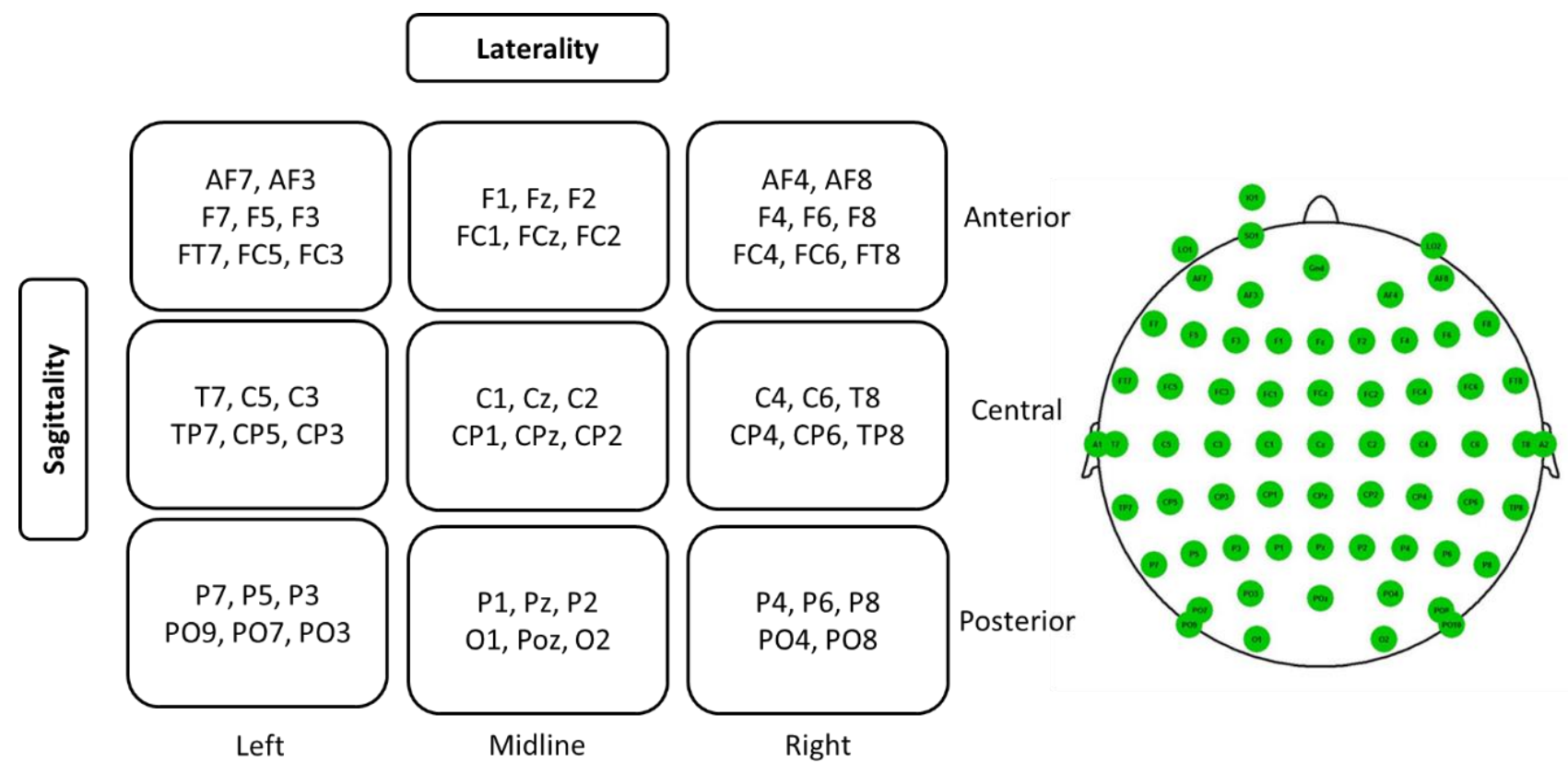

$550 \quad$ Figure 4.2 Grouping electrodes into regions of interest (ROI) by laterality and sagittality that remains consistent across analyses of Alpha spectral power and ERP components.

\section{SRTT Reaction time}

Participant RT performance for SRTT Familiarisation and Learning was analysed through linear mixed-effects regression (LMER) models using the lme4 package (Bates et al., 2015) in the R environment Ver. 3.4.3 (RStudio Team, 2015). The LMER allowed for controlling for variance associated with random factors to estimate group effects on response time performance. Another advantage of LMER over repeated measures analysis of variance (ANOVA), is the ability to account for nested (multiple observations in subject for single conditions) and crossed (single observation in subject with multiple conditions) and therefore reducing type 1 error rates (Boisgontier \& Cheval, 2016). In our main model, the outcome variable was - trial reaction time (ms) - and the factor variables: (1) Group (i.e. FAM21, FAM1 or Control), (2) Block, (3) Response error (i.e. whether trial was an error or not). Models were constructed separately for Familiarisation blocks (1 - 3) and Sequence learning blocks $(1-14)$. We specified subjects as a random factor to control for their associated intraclass correlation (i.e., random intercept models; Oberauer \& Kliegl, 2006) . Across all LMER models in this experiment, we present type II Wald chi square tests with KenwardRoger degrees of freedom approximation alongside the LMER results as this represents the level of confidence in the estimate of effects for each factorial comparison. 

components

571

The investigation of motor sequence learning indices in the form of general learning, sequence-specific learning and sequence interference performances provides an important dimension towards the understanding of behavioural performances changes. These indices are not addressed by LMER of RT performance and more importantly allows for comparison across other studies that assess rate of improvement, and sequence-specific learning based on transfer (Clegg et al., 1998; Du, Prashad, Schoenbrun, \& Clark, 2016). In our previous work we have investigated these indices and found that FAM practice provides performance benefits for general learning performance (Chan et al., In review) and sequence-specific learning (Chan et al., 2017). Our first goal was to understand differences in the performance of motor sequence learning indices. We also use a model-based approach to test the learning indices by entering them separately into the statistical models for the N200 and ERN components. The learning index that provided the best model fit represented by the lowest Akaike's Information Criterion (AIC) (Burnham \& Anderson, 2003), would subsequently be retained as a predictor for the ERP components and reported on.

To establish an index of general practice effects, we computed each participant's rate of performance improvement (i.e., reduction in RT) across SRTT Learning Blocks 1 - 12, when the SOC sequence was presented. We performed a regression analysis of correct-trial reaction times as the independent variable and trial number as a dependent variable using the Curvefit function in SPSS Ver. 24 (IBM Corp., Armonk, N.Y., USA). Our goal was to determine if a linear or quadratic model was more representative of a participant's learning style following a methodology established in Verstynen et al. (2012) and Chan et al. (In review), based the degree of significance for the beta coefficient. If the ANOVA $p$ value of the quadratic was more significant the beta coefficient for both the linear and quadratic model were summated, else the linear beta coefficient was accepted as is. The beta coefficient was considered the degree of trial RT change during learning and a representation of general learning effects during SRTT performance. Greater negativities in the values are representative of better general learning performance. It was established that general practice effects were representative of stimulus-based control (Chan et al., 2017, In review).

The sequence-specific learning percentage was calculated based on MRT performance between blocks 12 and 13. It was calculated by subtracting 1 from the ratio of block 13 MRT relative to block $12 \mathrm{MRT}$ and then multiplying by 100 . Increasing percentage values were 
602 indicative of greater levels of sequence-specific learning. We have previously found that 603 sequence-specific learning percentage was representative of plan-based control (Chan et al., 604 2017, In review). Sequence interference percentage was calculated by subtracting 1 from the 605 ratio of block 14 MRT relative to block $12 \mathrm{MRT}$ and then multiplying by 100 . Increased 606 percentages were an indication of increased interference effects.

\section{Event-related potentials: N200 and Error-related Negativity components data} 608 preparation and analysis

609 For the N200 component, EEG was stimulus-locked relative to the onset of each trial 610 presentation to create $1000 \mathrm{~ms}$ (millisecond) ERP epochs that consisted of -200 ms pre611 stimulus to $800 \mathrm{~ms}$ post-stimulus time window. Only the ERPs for correct trials for 612 Familiarisation and Sequence Learning Blocks were analysed. The Global Field Power 613 function in MNE-Python was then used to calculate the grand average waveform of all correct 614 trial epochs from Sequence Learning across channels for the 29 participants to determine the 615 N200 time window. Upon visual inspection of the grand average waveform, the average 616 amplitudes in the time window of $160-240$ ms was used to quantify the N200 component 617 (See Figure 4.3). This chosen time window was the same as previously reported by Moore et 618 al. (2012) for the quantification of the N200 component associated with attentional effects 619 after FAM training. Electrodes and their averaged amplitudes were also grouped into the nine 620 ROIs shown in Figure 4.2, to understand the N200 amplitude differences based on scalp topography. 


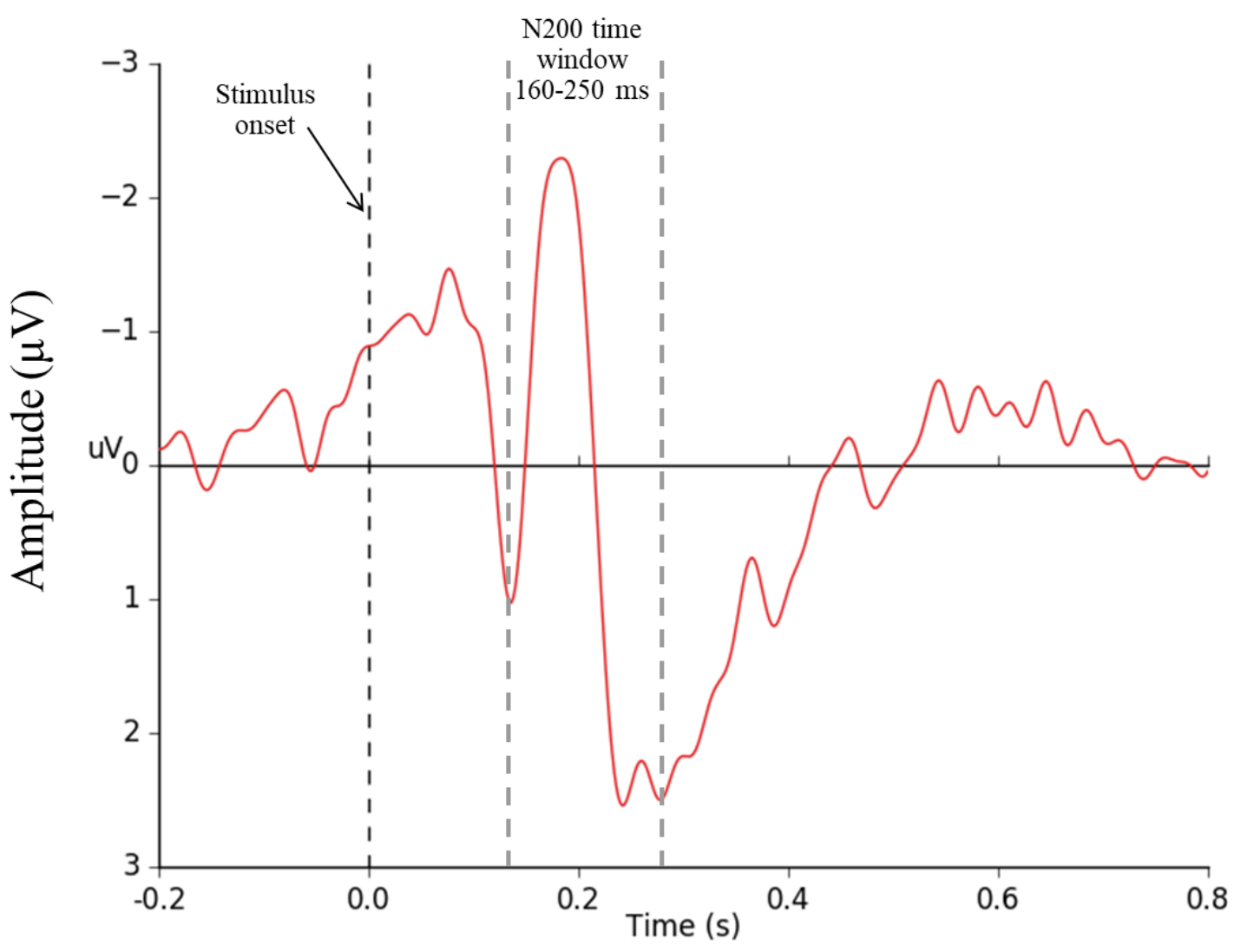

Figure 4.3 Global field power of all EEG channels for stimulus-locked correct trials during sequence learning. The time window of $160-240 \mathrm{~ms}$ was used to quantify the N200 time window for analysis.

We then analysed the amplitude in the N200 time window with LMER models using the lme4 package (Bates et al., 2015) in the R environment. Models were constructed separately for Familiarisation and Sequence Learning N200. For the Familiarisation N200 model, the outcome variable was - mean correct trial epoch amplitude $(\mu \mathrm{V})$ and the factor variables: (1) Group (i.e. FAM21, FAM1 or Control), (2) Laterality, (3) Sagittality. In addition, for the Sequence Learning N200 model, each Sequence Learning index (general learning effects, sequence-specific learning, interference) was tested separately to model the relationship of N200 with learning indices (based on AIC estimates, only the index that provides the best fit will be reported). Subjects was specified as a random factor to control for their associated intraclass correlation (i.e., random intercept models; Oberauer \& Kliegl, 2006).

For the ERN component, EEG was response-locked relative to the onset of each participant's trial response in SRTT task to create $1000 \mathrm{~ms}$ epochs that consisted of $-200 \mathrm{~ms}$ pre-response to $800 \mathrm{~ms}$ post response time window. Separate averaged waveforms were 
640 computed for error trials (ERN) and correct trials (correct-related negativity or CRN) in using 641 the Global Field Power function in MNE-Python for all trial epochs using the typically 642 reported $\mathrm{Cz}$ channel (Beaulieu et al., 2014) for the 29 participants . Based on the grand 643 average waveform, averaged amplitudes in the time window of $0-150 \mathrm{~ms}$ was used to 644 quantify ERN and CRN components (See Figure 4.3). This chosen time window was the 645 same as previously reported by Teper \& Inzlicht (2013) for the quantification of the ERN to 646 investigate the effects from FAM.

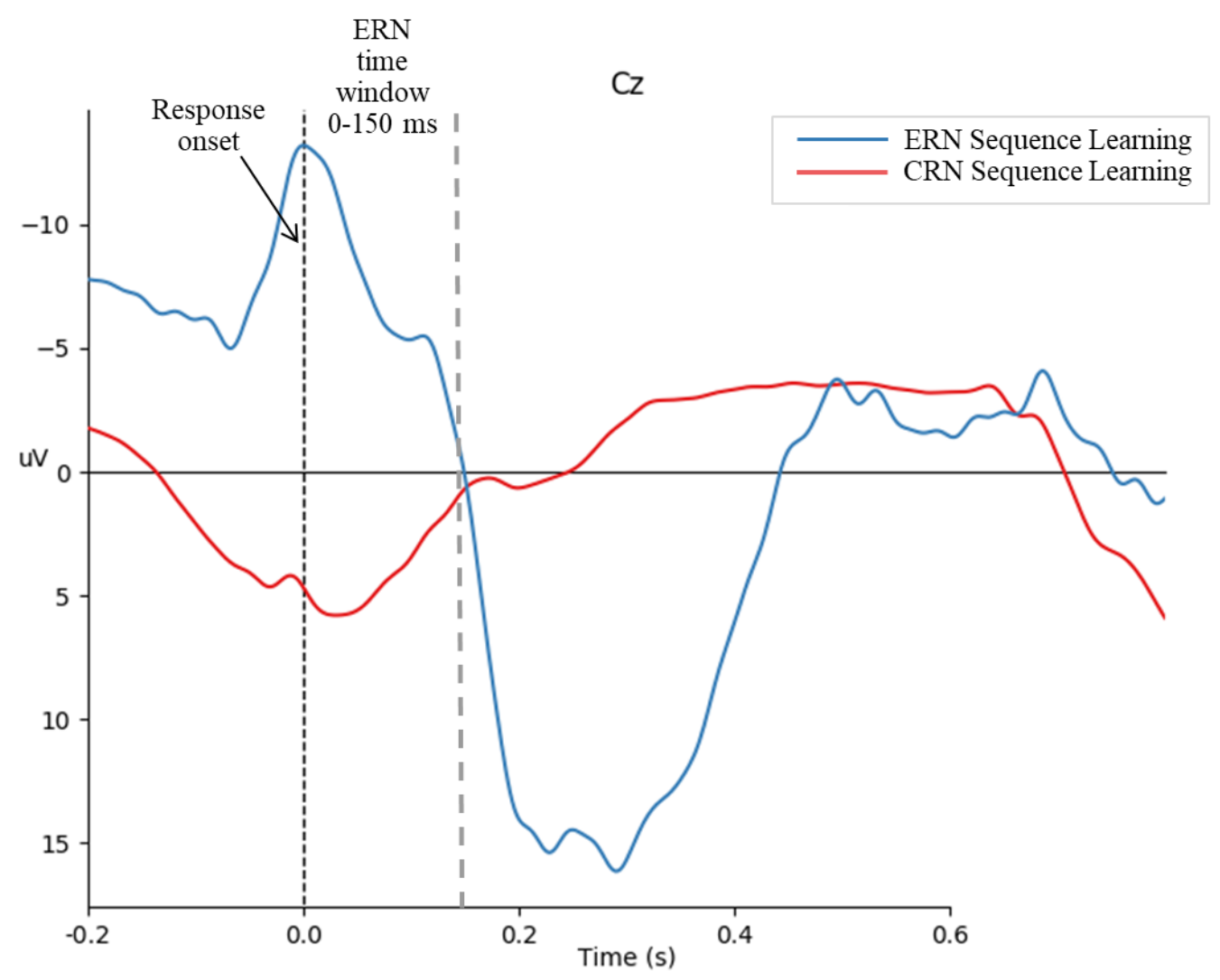

648 Figure 4.4 Global field power for Cz channel for response-locked error and correct trials

649 during sequence learning. The time window of 0 - 150 ms was used to quantify the ERN time 650 window for analysis.

The elicited averaged neurophysiological brain activity for all correct trials was subtract from that of all error trials across Sequence Learning (i.e., mean ERN-CRN). This results in the final mean ERN component amplitude for each channel, for each participant is then analysed. Typically, the ERN is measured over a single channel or a small number of channels in the central region of the scalp (e.g. FCz/Cz) (Beaulieu et al., 2014; Larson et al., 2013) due to likely contributions of the anterior cingulate cortex as a neural generator for the ERN (Holroyd \& Coles, 2002). In the current experiment, we took the opportunity to 
658 understand the dynamics of the ERN over the entire scalp instead of processing just individual

659 channels or grouping central EEG channels and grouped electrodes by nine ROIs segregated

660 by sagittality and laterality (Figure 4.2).

661 We then analysed the amplitude for the ERN component with LMER whereby models 662 were constructed separately for Familiarisation ERN and Sequence Learning ERN. For the 663 Familiarisation ERN model, the outcome variable was - mean ERN amplitude $(\mu \mathrm{V})$ and the 664 factor variables: (1) Group (i.e. FAM21, FAM1 or Control), (2) Laterality, (3) Sagittality. In 665 addition, for the Sequence Learning ERN model, each Sequence Learning index was added 666 separately to model the relationship of ERN with learning indices (based on AIC estimates, 667 only the index that provides the best fit will be reported). We specified subjects as a random 668 factor to control for their associated intraclass correlation.

\section{Results}

670 Cognitive task related spectral power in the Alpha band (8-13Hz)

671 The LMER model analysis of alpha spectral power revealed no significant main effect of

672 Group $(p=.16)$, significant Group x Sagitality interaction, $\chi^{2}(4, \mathrm{~N}=29)=58.5, p<.001$, a 673 significant Group x Laterality interaction, $\chi^{2}(4, \mathrm{~N}=29)=39.0, p<.001$, a, and a significant 674 Group x Laterality x Sagitality interaction, $\chi^{2}(8, N=29)=28.2, p<.001$. In general, alpha 675 spectral power was higher in Control compared to FAM1 and FAM21 across central-midline, 676 with greatest differences in posterior (midline and right) regions (see Figure 4.7). FAM21 and FAM1 was not different across all ROIs during FAM practice. 


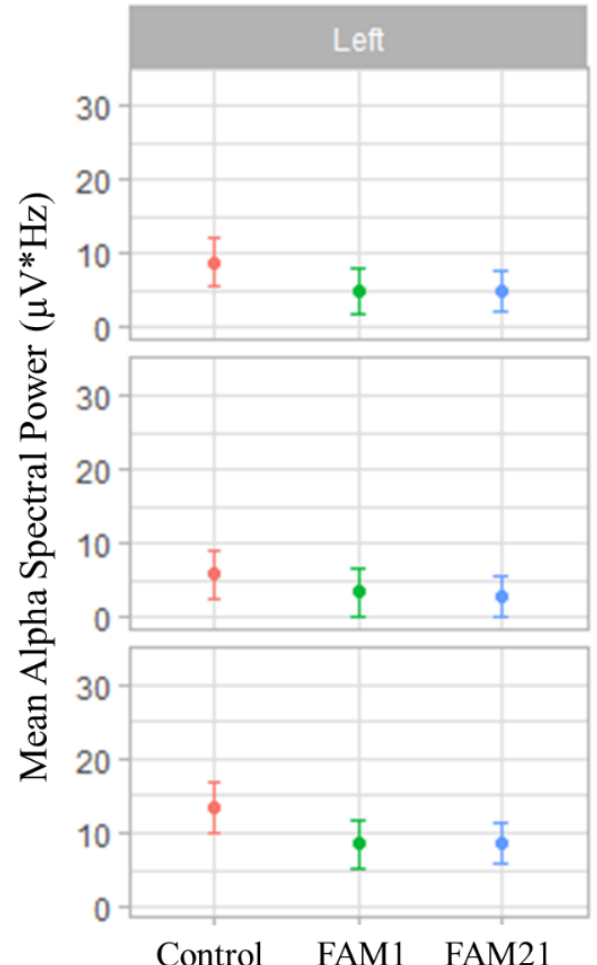

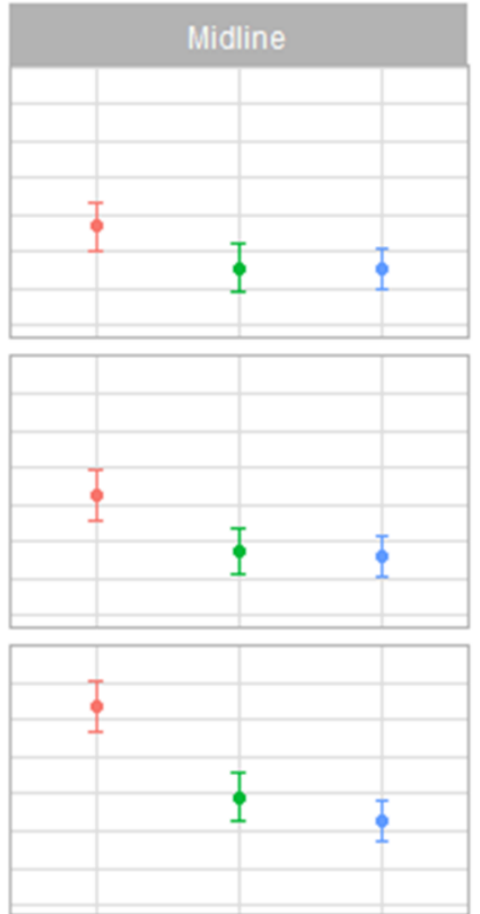

Control FAM1 FAM21
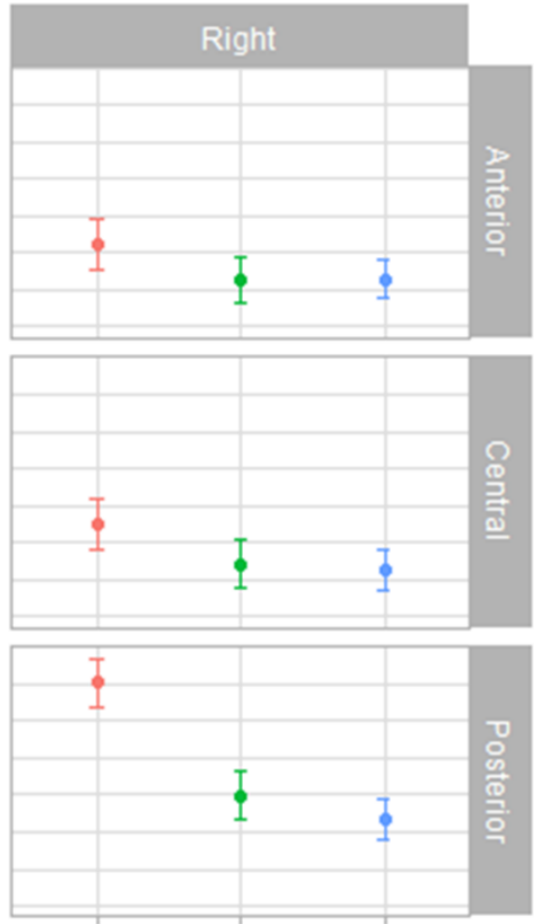

Control FAM1 FAM21
678

679

680

681

682

683

684

685

686

687

688

689

690

691

692

693

694

695

696

Figure 4.5 Alpha $(8-13 \mathrm{~Hz})$ spectral power effects across regions of interest (ROI) for the $21^{\text {st }}$ session of cognitive training performed in the laboratory prior to sequence learning. The FAM21 group completed 20 sessions of focused attention meditation (FAM) prior daily, and in this session performed a single FAM practice. FAM1 group completed 20 sessions of an audio listening task (LT) prior daily, and in this session performed a single FAM practice. The Control group completed 20 sessions of an audio-listening task prior daily, and in this session performed a single LT practice. Error bars represent standard error of the mean.

\section{SRTT Reaction time performance: Familiarisation}

Firstly, the initial LMER model analysis of Familiarisation (blocks 1 - 3) performance revealed that there was neither a significant Group x Response error interaction for trial RT ( $p$ $=.20)$, nor a significant Group x Response error x Block interaction for trial RT $(p=.50)$. Accordingly, the model was simplified by removing all incorrect trials. This resulted in the exclusion of $2.6 \%$ of all SRTT trials in Familiarisation from further analysis.

For the main Familiarisation LMER model with remaining correct trials revealed no significant main effect of Group $(p=.65)$, a significant effect of Block, $\chi^{2}(2, N=29)=112.1$, $p<.001$, reflecting shorter RT across familiarisation blocks, particularly from the first to second blocks. In addition, a significant Group x Block interaction, $\chi^{2}(4, \mathrm{~N}=29)=20.1, p<$ .001 , showed that the relationship between blocks and trial RT changed as a function of 
697

698

699

700

701

702

703

704

705

706

707

708

709

710

Group. The interaction was predicated in block 1 of Familiarisation where the FAM21 group was performing faster than FAM1 and Control groups. Subsequent blocks 2 and 3 showed that trial RT performance was equal amongst the groups and converged (see Figure 4.6).
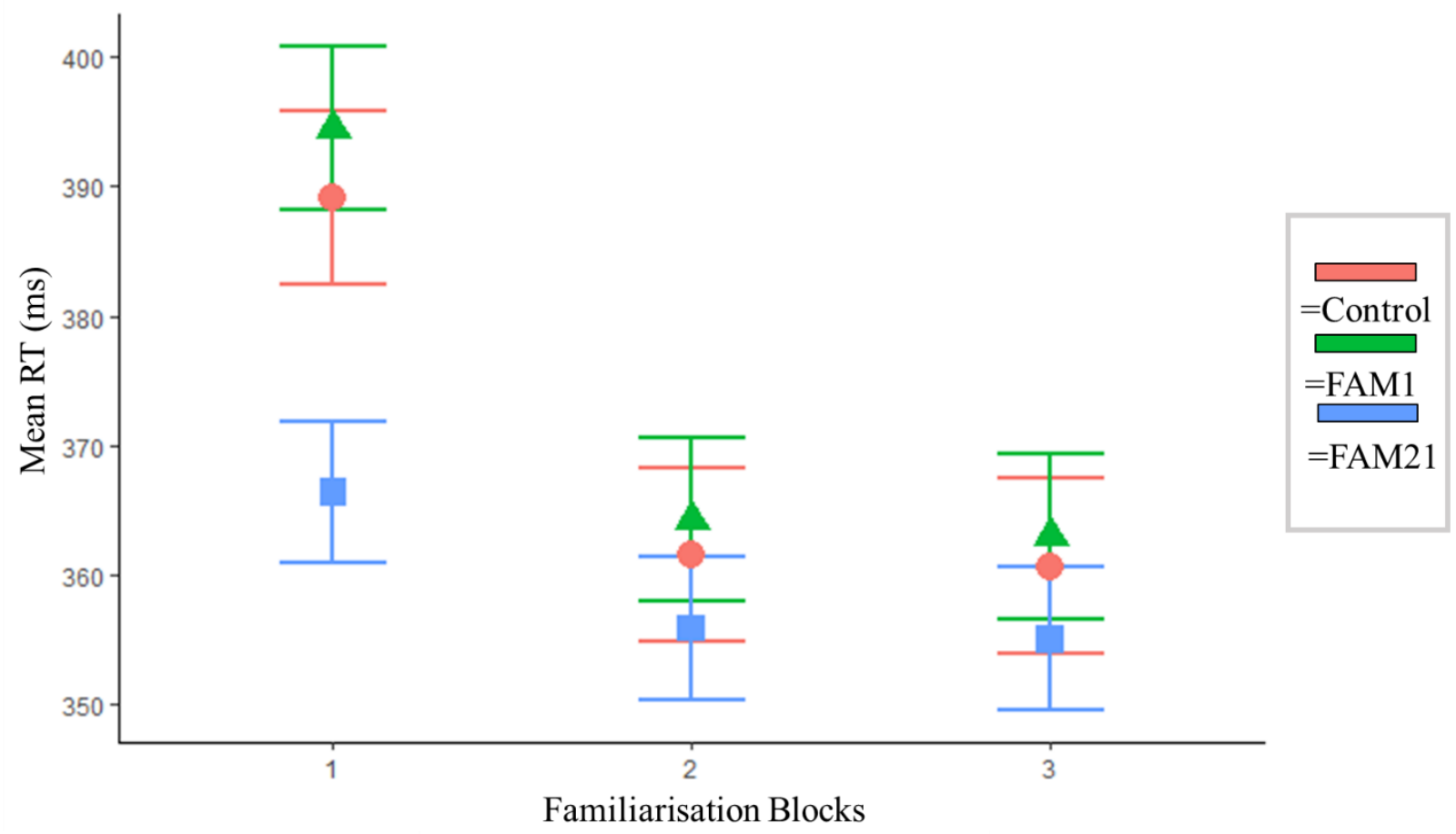

Figure 4.6 Familiarisation Mean RT: Group x Block Interaction. Modelled effects in Familiarisation blocks $1-3$ of the serial reaction time task (SRTT) involving pseudo-random ordered trials. Familiarisation were performed prior to any experimental manipulation. Familiarisation blocks 1 showed trial RT difference between the groups but in blocks 2 and 3, a convergence of performance was evident across all groups. Error bars represent $95 \%$ confidence intervals.

\section{SRTT Reaction time performance: Sequence Learning}

The initial LMER model analysis of Sequence Learning (blocks 1-14) performance revealed that there was no significant Group x Response error interaction for trial RT ( $p=.93)$, nor was there a significant Group x Response error x Block interaction for trial RT $(p=.06)$. The linear mixed model was thus simplified by removing all erroneous trials and Response error as a factor for further analysis of RT performance. This resulted in the exclusion of $3.4 \%$ of all SRTT trials in sequence Learning Blocks.

The Sequence Learning LMER for the remaining correct trials revealed no significant main effect of Group $(p=.79)$ and a significant effect of Block on trial RT, $\chi^{2}(13, \mathrm{~N}=29)=$ 
3991.9, $p<.001$, where all groups were showing RT reductions across Learning Blocks $1-$ 12, an increase in RT for Transfer Block 13 and then reduction in RT for Block 14. This RT pattern was typical of SRTT performance (Robertson, 2007; Song, Howard, \& Howard, 2008). There was a significant Group $x$ Block interaction, $\chi^{2}(26, \mathrm{~N}=26)=234.9, p<.001$, such that the groups demonstrated differences in RT performance across blocks. The following are the effects revealed by the plots of the LMER for Sequence Learning. In block 1, FAM21 had a shorter RT compared to FAM1 but not Control. FAM1 and Control had similar RT. In block 2, FAM21 had shorter RT compared to both FAM1 and Control. In block 3, FAM21 had shorter RT compared to FAM1 but not control. FAM1 and Control had similar performance. In blocks 4 and 5, FAM21 had faster RT compared to both FAM1 and Control, whereby both groups were not different. In block 6 and 7, both FAM21 and Control were not different but had shorter RTs compared to FAM1. In block 8, FAM21 had the shortest RT whilst FAM1 and Control had longer RTs and were not different from each other. In block 9, FAM21 had the shortest RT but was not different from FAM1. Both FAM21 and FAM1 had shorter RTs than Control. In block 10, FAM21 has the shortest RT and was different from Control but not different from FAM1. FAM1 was not different from Control. In block 11, FAM21 had the shortest RT and was different from Control but not different from FAM1. FAM1 had a shorter RT compared to Control. In pre-transfer block 12, although FAM1 had the shortest RT, all groups were not different from each other. In transfer block 13, FAM21 had the shortest RT and was different from Control. FAM1 was not different from FAM21 or Control, which had the longest RT. In final block 14, all groups were performing similarly.

As a summary of the Sequence Learning performance, the FAM21 group demonstrated shortest RT performance in 11 of 14 blocks, FAM1 only in one block and Control for none of the blocks. Specifically, the effects showed that FAM21 had shorter RTs compared to Control for 8 of 14 blocks (2, 4, 5, 8, 9, 10, 11 and 13), and had shorter RTs compared to FAM1 on 8 of 14 blocks $(1,2,3,4,5,6,7$ and 8). FAM1 had shorter RTs than Control in 2 of 14 blocks (9 and 11). Control had shorter RTs than FAM1 in 2 of the 14 blocks (6 and 7). Neither FAM1 nor Control had shorter RTs compared to FAM21 in any of the Sequence Learning blocks (please see Figure 4.7 for effects plot of the Sequence Learning LMER). 


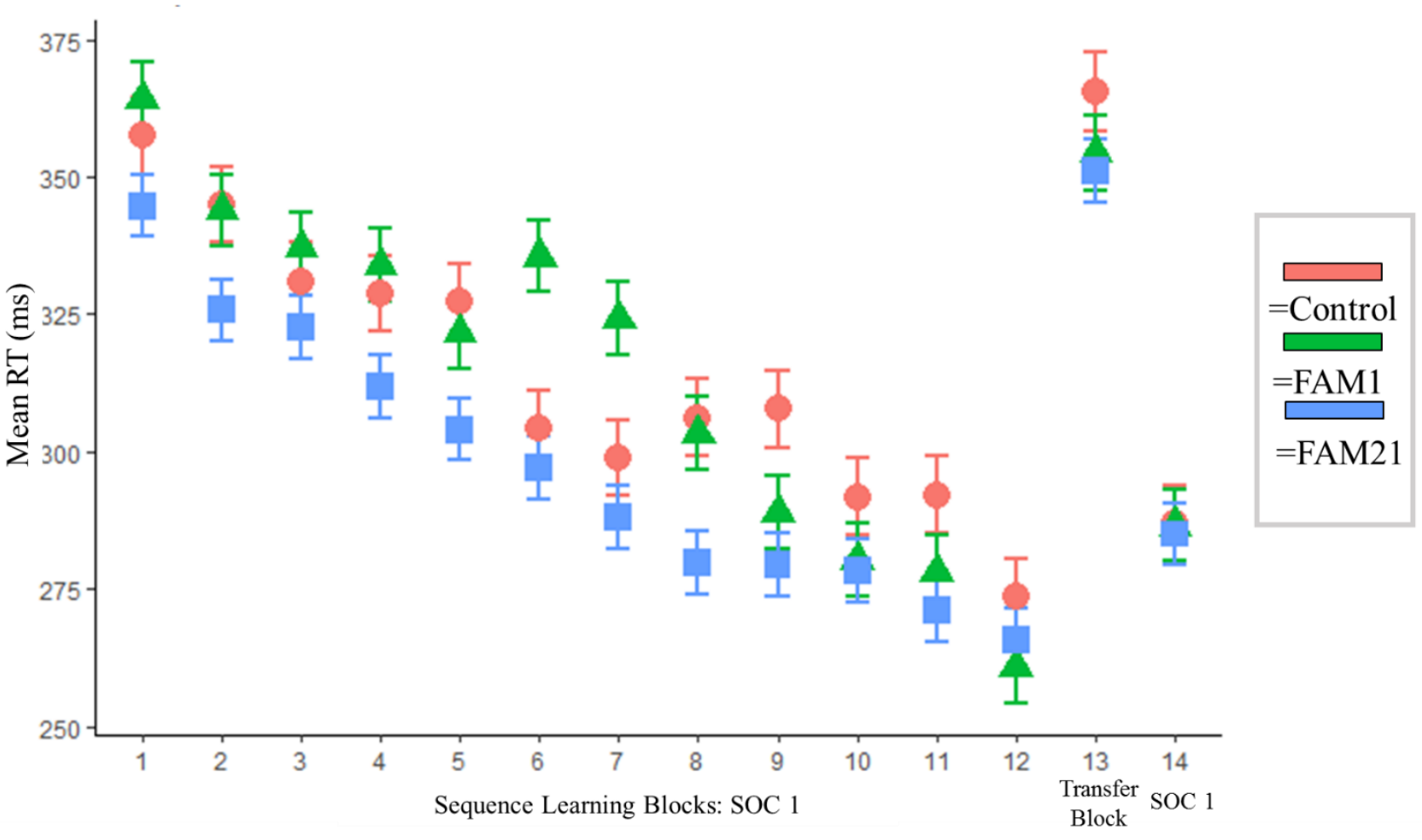

Figure 4.7 Sequence Learning Mean RT: Group x Block interaction. Modelled effects in Sequence Learning blocks of the Serial Reaction Time Task (SRTT) involving second-ordered conditional (SOC) Sequence Learning blocks 1-12, a pseudo-random ordered Transfer block 13, and reinstatement of the learned SOC sequence in block 14. The FAM21 group completed 20 sessions of focused attention meditation (FAM) and a single FAM session immediately prior to the SRTT. FAM1 group completed 20 sessions of an audio-listening task and a single FAM session immediately prior to SRTT. The Control group completed 20 sessions of an audio-listening task and a single-session of audio-listening task immediately prior to the SRTT. Error bars represent $95 \%$ confidence intervals.

\section{Event-related potential: $\mathbf{N 2 0 0}$ component}

Because no significant Group x Response interactions in the both familiarisation and sequence learning in behavioural RT were observed, we continued to analyse the amplitudes of only correct trial epochs in the N200 time window and sought to understand if it served as an index of top-down cognitive control activation during sequence learning.

\section{Familiarisation N200}

The results of the Familiarisation LMER for the N200 component amplitude revealed no significant main effect of Group $(p=.17)$, a significant Group x Laterality interaction, $\chi^{2}(4$, 


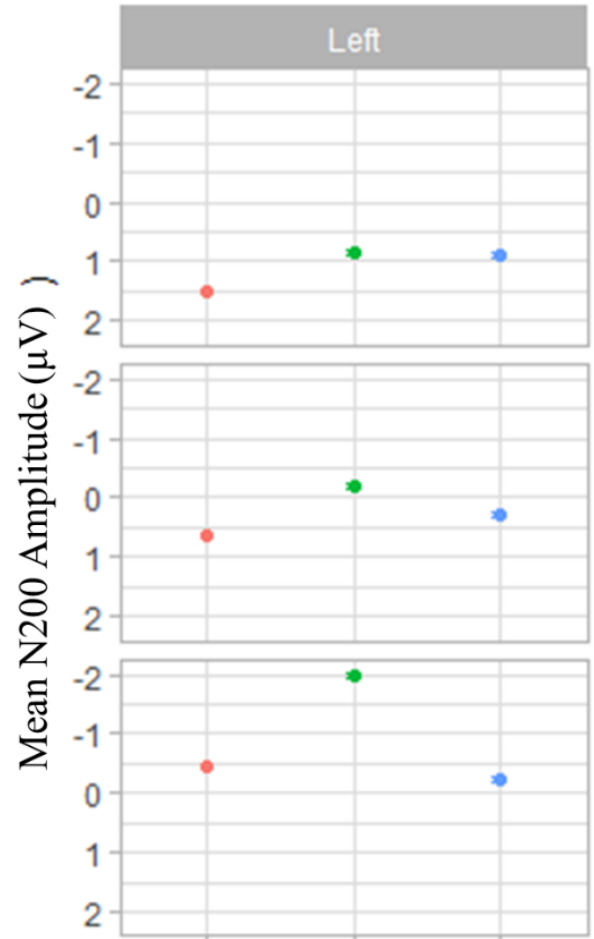

Control FAM1 FAM21

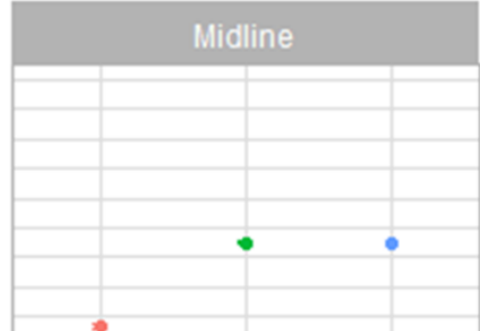

$\rightarrow$

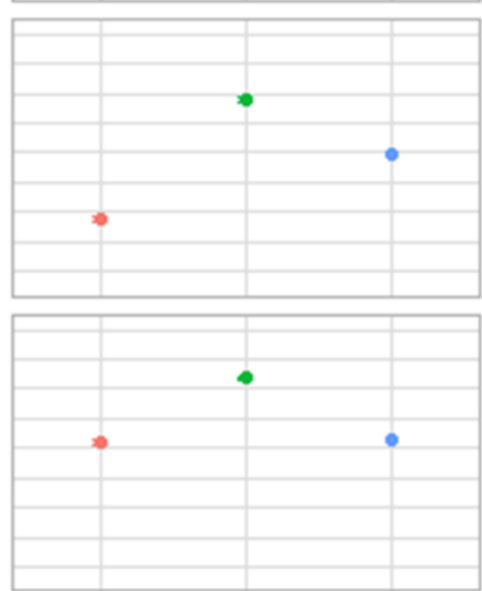

Control FAM1 FAM21
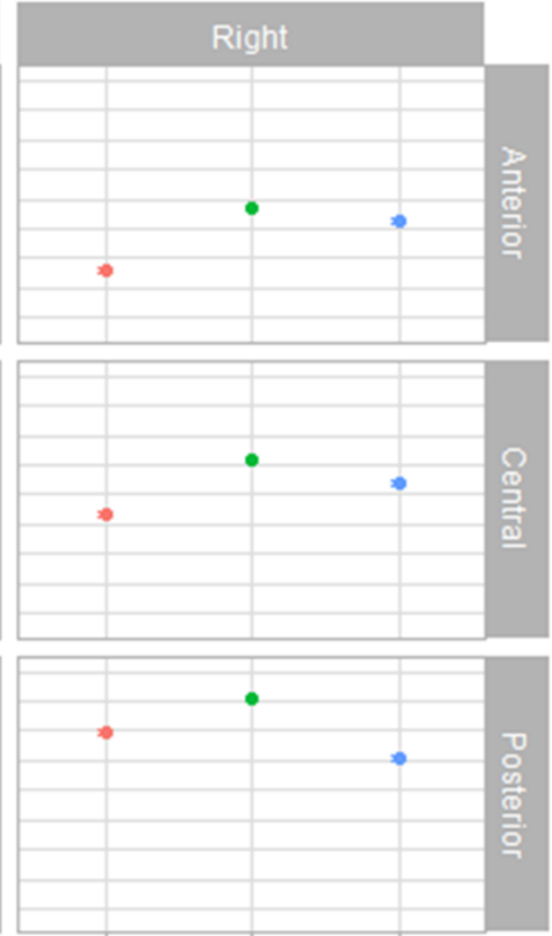

Control FAM1 FAM21

Figure 4.8 Familiarisation N200 Amplitude: Group x Laterality x Sagittality Interaction. Modelled effects for the N200 component stimulus-locked to SRTT trial onset for all correct trials performed across Familiarisation blocks. Although groups are indicated, familiarisation was performed prior to any experimental manipulation. Error bars represent standard error of the mean, in this case are very small due to high consistency from selecting correct responses. 
783

784

785

786

787

788

789

790

791

792

793

794

795

796

797

798

799

800

801

802

803

804

805

\section{Sequence learning N200}

The model-based approach for determining the sequence index as a predictor for N200 component amplitude revealed that general learning provided the best model fit (AIC = 13625965) compared to sequence-specific learning $(\mathrm{AIC}=13626009)$ and interference (AIC $=13641071)$. Therefore, the reported model included general practice effects as a factor in the Sequence Learning LMER for the N200 component amplitude. The results yielded a significant main and interaction effects in the model and are reported in Table 1.

Table 4.1 Overall sequence learning main and interaction effects on mean EEG amplitude for the N200 component for correct trials. General learning performance provided the best model fit and therefore retained as a predictor for N200 amplitudes.

\begin{tabular}{lccc}
\hline Effect & df & $\mathbf{X}^{\mathbf{2}}$ & Significance \\
\hline Group & 2 & 14.90 & $* * *$ \\
Group x General learning & 2 & 7.26 & $*$ \\
Group x Sagittality & 4 & 5243.61 & $* * *$ \\
Group x Laterality & 4 & 2749.78 & $* * *$ \\
Group x Sagittality x General learning & 4 & 8551.15 & $* * *$ \\
Group x Laterality x General learning & 4 & 2690.61 & $* * *$ \\
Group x Sagittality x Laterality & 8 & 1007.27 & $* * *$ \\
Group x Sagittality x Laterality x General learning & 8 & 361.52 & $* * *$ \\
\hline
\end{tabular}
Note. ${ }^{*} p<.05, * * p<.01, * * * p<.001$

We report on the significant Group x Laterality $x$ Sagittality interaction as a way to firstly understand the differences in Sequence Learning from Familiarisation. In the anterior (left, midline and right) ROIs, mean amplitude between the groups appeared to be in a gradient manner. Increased negativity was observed in FAM21 compared to FAM1 and Control. FAM1 also had greater negativities in same ROIs compared to Control which had the smallest negative deflection. A similar pattern was observed in the central (left, midline and right) ROIs, whereby FAM21 had the greatest negative deflection and Control with the smallest negative deflection. FAM1 amplitude was also in between the two other groups. In the posterior-left, FAM1 had the greatest negative deflection, followed by FAM21 and Control. In the posterior-midline, FAM21 had the greatest negativities and this was different from FAM1 and Control. FAM1 had greater negativity compared to Control. Lastly, in the posterior-right ROI, FAM21 still had the greatest negativity compared to FAM1 and Control. 
806 FAM1 and Control were not different. Please see Figure 4.9 for in modelled interaction 807 effects.

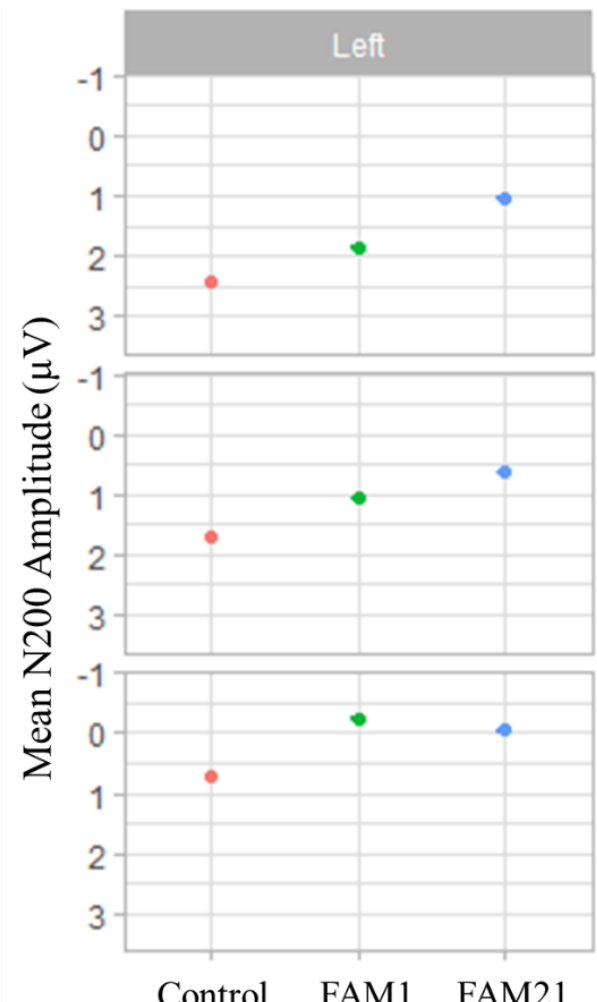

Control FAM1 FAM21
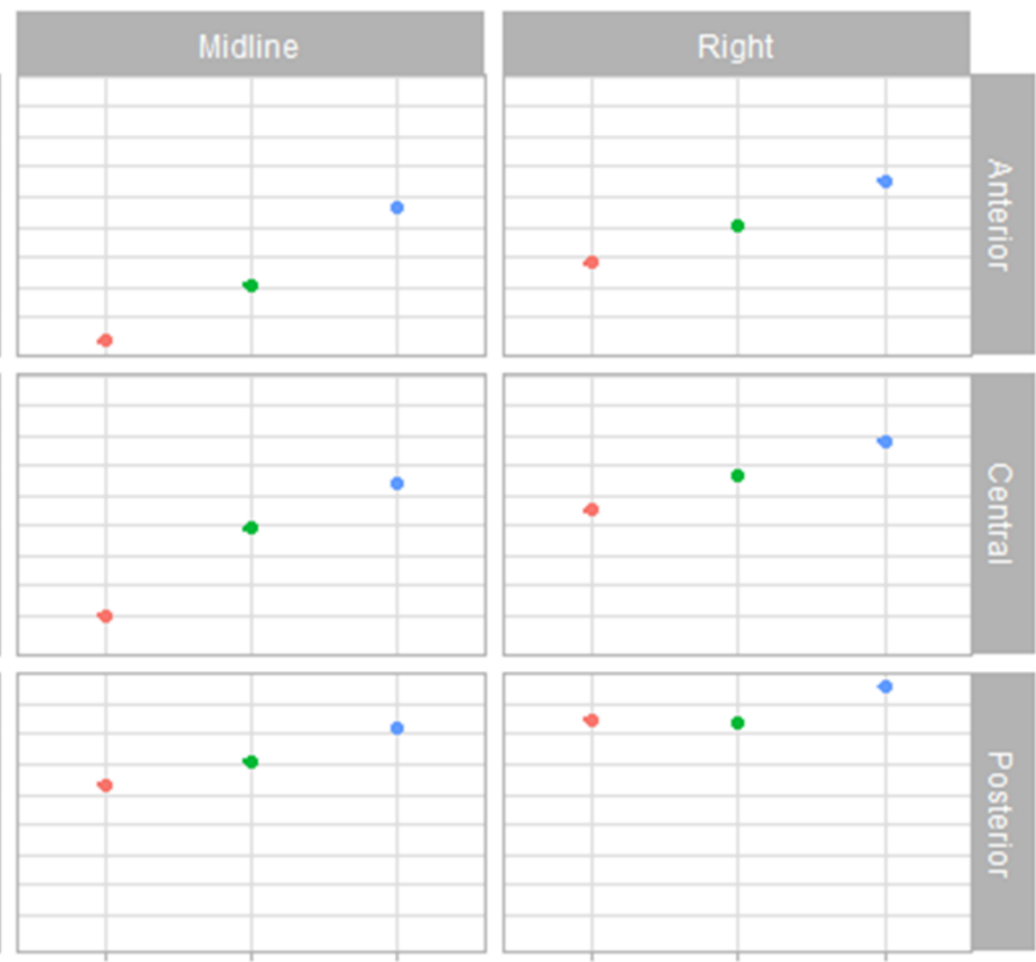

Control FAM1 FAM21

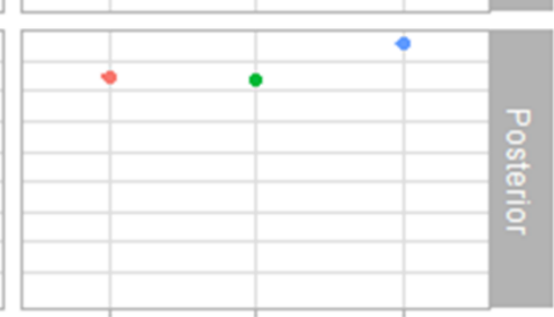

Control FAM1 FAM21
808

Figure 4.9 Sequence Learning N200 Amplitude: Group x Laterality x Sagittality Interaction. Modelled effects for the N200 component stimulus-locked to SRTT trial onset for all correct trials performed during Sequence Learning blocks. The FAM21 group had completed 20 sessions of focused attention meditation (FAM) and a single FAM session immediately prior to the SRTT. FAM1 group completed 20 sessions of an audio-listening task (LT) and a single FAM session immediately prior to SRTT. The Control group completed 20 sessions of an LT and a single-session of LT immediately prior to the SRTT. Error bars represent standard error of the mean, in this case are very small due to consistency from selecting correct responses.

We also report on the significant Group x Laterality x Sagittality x General learning interaction as a way to understand the effects between cognitive training, general learning performance and N200 amplitude in the different regions. The most pronounced effects in the FAM21 group were observed in the anterior (left and midline) and central (left and midline) ROI. The pattern that emerged revealed that for participants in FAM21, at poorer levels of general learning performance had greater N200 negativities compared to other groups to 
maintain performance. However, as general learning performance increased, FAM21 had the greatest rate of reduction in N200 amplitude compared to FAM1 and Control. At very high levels of general learning performance, FAM21 group had N200 amplitudes that were less negative compared to FAM1 and Control.
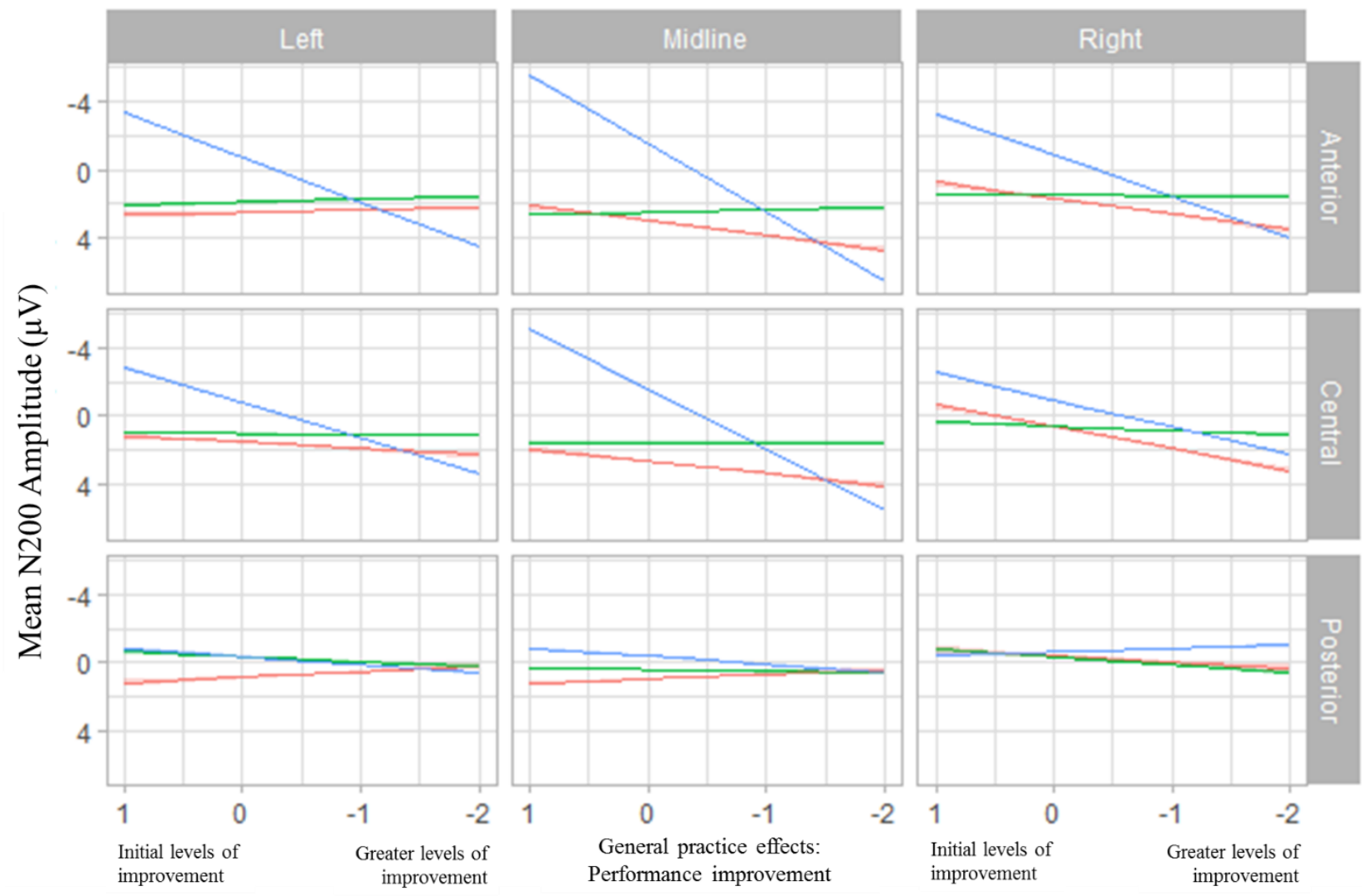

Figure 4.10 Sequence Learning N200 Amplitude: Group x Laterality $x$ Sagittality $x$ General practice effects Interaction. Modelled effects for the N200 component stimulus-locked to SRTT trial onset for all correct trials performed during Sequence Learning blocks against general practice effects. The FAM21 group completed 20 sessions of focused attention meditation (FAM) and a single FAM session immediately prior to the SRTT. FAM1 group completed 20 sessions of an audio listening task (LT) and a single FAM session immediately prior to SRTT. The Control group completed 20 sessions of LT and a single-session of LT immediately prior to the SRTT. Filled colours represent $95 \%$ confidence intervals, although very small due to high level of consistencies for correct trials. Effects are most pronounced across anterior and central regions of interest (ROIs). The trends show that FAM21 had greater levels of top-down activation to support initial levels of improvement compared to FAM1 and Control. As levels of improvement start to increase, FAM21 have far greater decline of amplitudes compared to FAM1 and Control to support general practice effects. 
842

843

844

845

846

847

848

849

850

851

852

853

\section{Event-related potential: Error-related Negativity (ERN)}

The results of the Familiarisation LMER for the ERN component revealed no significant main effect of Group ( $p=.96)$, a significant Group x Sagitality interaction, $\chi^{2}(4, N=29)=43.2, p<$ .001 , no significant Group x Laterality interaction $(p=.65)$, and no Group x Laterality $\mathrm{x}$ Sagitality interaction $(p=.52)$. The plots for the effects in ERN amplitude showed that in anterior ROI, FAM21 and Control were similar and had greater ERN negativities compared to FAM1. In the central ROI, FAM21 had a greater ERN negativity compared to FAM1 and Control, where they were both similar. In the posterior ROI, both FAM1 and Control were similar and had greater ERN negativities compared to FAM21. It should be noted that largest negative deflections amongst groups were found in the central region suggestive of central channels being significant contributors to the ERN that in line with literature (Botvinick et al., 2001; Carter et al., 1998; Larson et al., 2013).

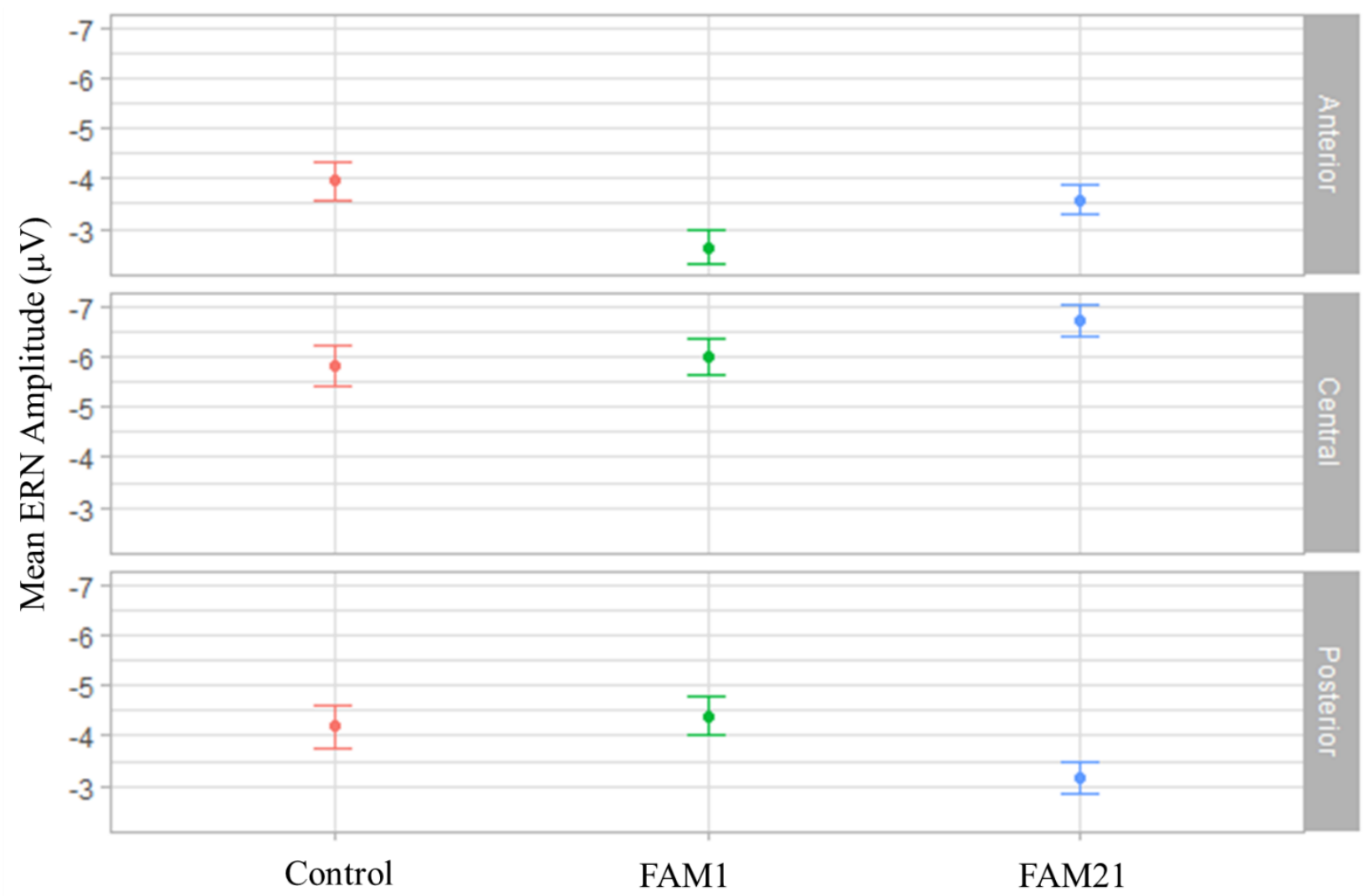

Figure 4.11 Familiarisation ERN Amplitude: Group x Sagittality Interaction. Modelled effects for response-locked ERN component across Familiarisation blocks. Although groups are indicated, familiarisation was performed prior to any experimental manipulation. Error bars represent standard error of the mean. 
The model-based approach for determining the sequence index as a predictor for ERN component amplitude revealed that general learning also provided the best model fit (AIC $=$ 8393.6) compared to sequence-specific learning $(\mathrm{AIC}=8605.1)$ and interference $(\mathrm{AIC}=$ 8577.3). Therefore, the reported model included general learning as a factor in the Sequence Learning LMER for the ERN component amplitude. The results in the model yielded only a significant Group x Sagitality interaction, $\chi^{2}(4, \mathrm{~N}=29)=41.5, \mathrm{p}<.001$, and a significant

865 Group $x$ Laterality interaction, $\chi^{2}(4, N=29)=13.1, p<.05$. The remainder of the effects are 866 reported in Table 4.2.

Table 4.2 Sequence Learning main and interaction effects of mean EEG amplitude for the ERN component. General learning performance provided the best model fit and therefore retained as a predictor for ERN amplitudes.

\begin{tabular}{lccc}
\hline Effect & df & $\mathbf{X}^{\mathbf{2}}$ & Significance \\
\hline Group & 2 & 3.56 & \\
Group x General learning & 2 & 0.58 & \\
Group x Sagittality & 4 & 41.48 & $* * *$ \\
Group x Laterality & 4 & 13.12 & $*$ \\
Group x Sagittality x General learning & 4 & 3.21 & \\
Group x Laterality x General learning & 4 & 5.30 & \\
Group x Sagitality x Laterality & 8 & 10.79 & \\
Group x Sagitality x Laterality x General learning & 8 & 8.65 & \\
\hline
\end{tabular}
Note. ${ }^{*} p<.05, * * p<.01, * * * p<.001$ showed that in the anterior ROI, FAM1 had greater ERN negativities compared to FAM21 and Control. FAM21 had greater negativity compared to Control. In the central ROI, both FAM21 and FAM1 were not different from each other but had greater ERN negativities compared to Control. In the posterior ROI, FAM1 had the greatest ERN negativity compared to FAM21 and Control, where their amplitudes were similar. 


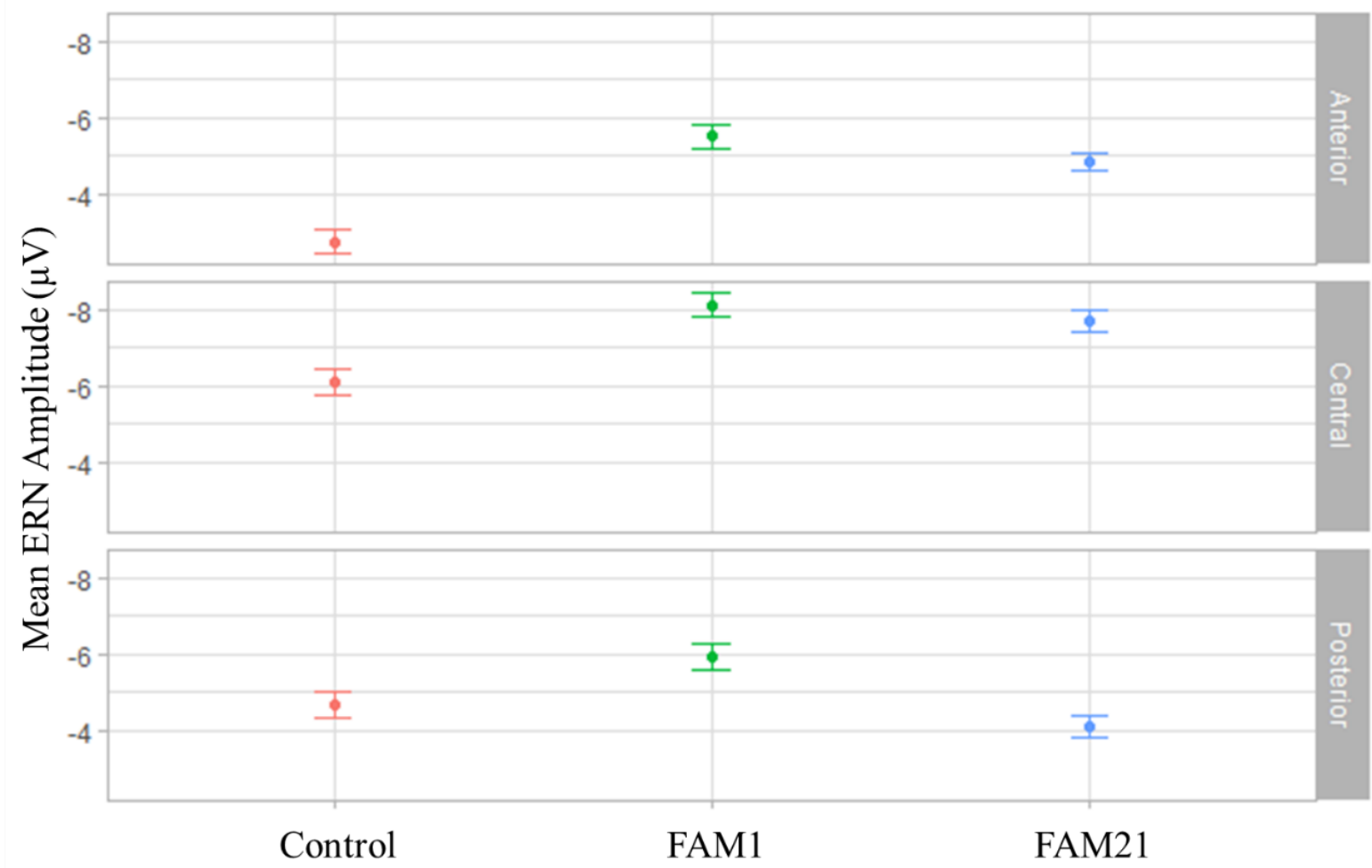

878 Figure 4.12 Sequence ERN Amplitude: Group x Sagittality Interaction. Modelled effects for response-locked ERN component across Sequence Learning blocks. The FAM21 group completed 20 sessions of focused attention meditation (FAM) and a single FAM session

881 immediately prior to the SRTT. FAM1 group completed 20 sessions of an audio-listening task 882 (LT) and a single FAM session immediately prior to SRTT. The Control group completed 20 883 sessions of LT and a single-session of LT immediately prior to the SRTT. Error bars represent standard error of the mean. showed a clear and similar pattern across the left, midline and right ROIs. All plots show that FAM1 had the greatest negativities, with FAM21 the next greatest negativities and then 888 Control with the least negativities for ERN amplitudes. It should be noted that midline ROI 889 had the greatest level of negativities in the ERN compared to left and right ROIs. 

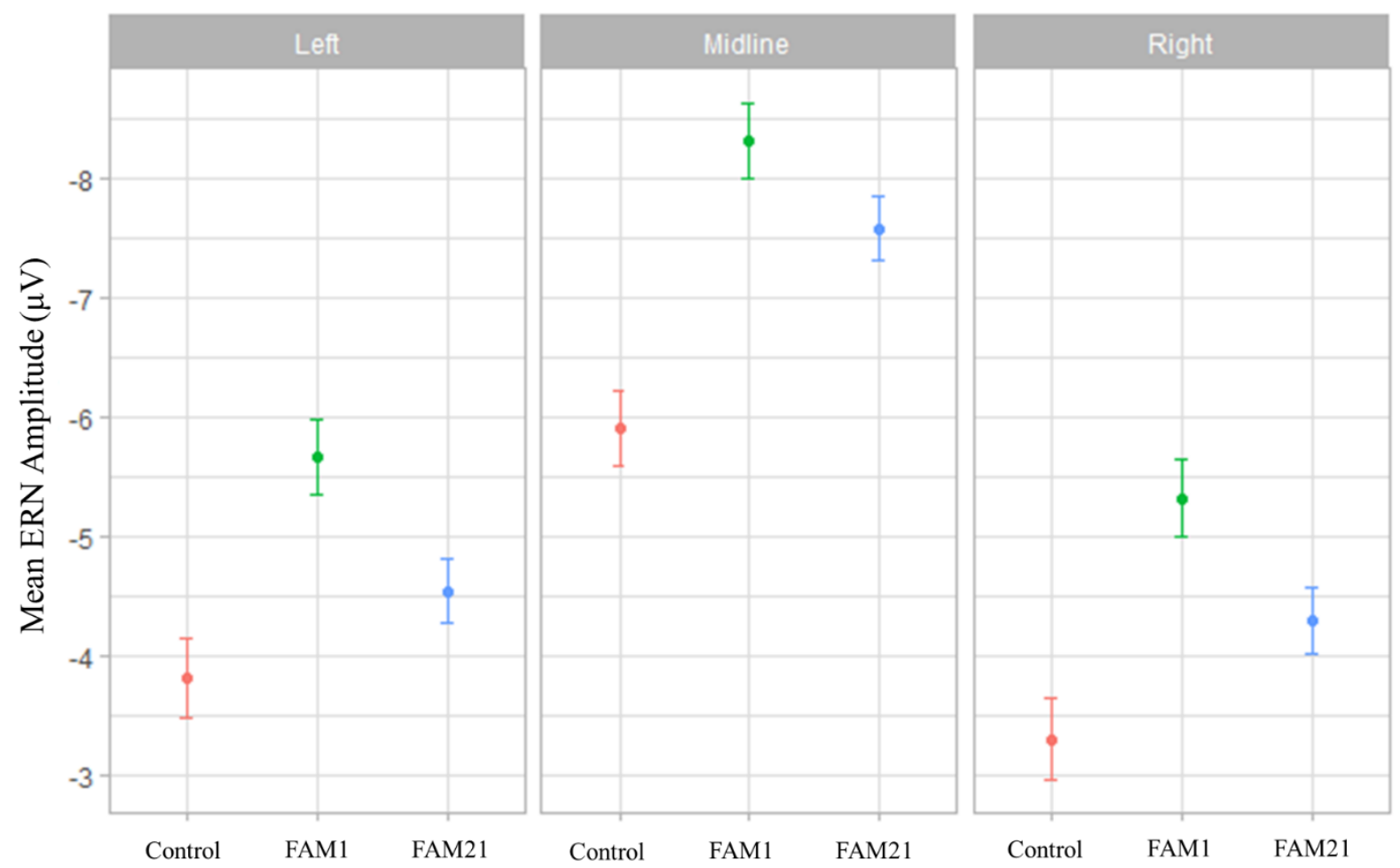

Figure 4.13 Sequence ERN Amplitude: Group x Laterality Interaction. Modelled effects for response-locked ERN component across Sequence Learning blocks. The FAM21 group completed 20 sessions of focused attention meditation (FAM) and a single FAM session immediately prior to the SRTT. FAM1 group completed 20 sessions of an audio-listening task (LT) and a single FAM session immediately prior to SRTT. The Control group completed 20 sessions of LT and a single-session of LT immediately prior to the SRTT. Error bars represent standard error of the mean.

\section{Discussion}

Previous research has demonstrated that a single-session of FAM strengthens top-down control which transfers to subsequent sequence learning resulting in increased bias towards stimulus-based modes of sequential action (Chan et al., 2017, In review; Immink et al., 2017). The current experiment aimed to test if brief FAM training would further enhanced top-down cognitive control effects of a single-session of FAM for subsequent motor sequence learning. Guided by the overlapping evidence between FAM effects and motor sequence learning (Moore et al., 2012), the N200 component is considered highly sensitive to cognitive control and therefore we employed the N200 ERP component as an index of top-down during sequence learning. We predicted that general practice effects from sequence learning would be representative of stimulus-based control and reflected by changes in N200 amplitudes. 
Specifically, if short-term FAM training increased the degree of stimulus-based control in comparison to single-session FAM, we predicted that the N200 amplitude during sequence learning would also be further enhanced.

We also sought to investigate if the ERN component, that is closely related to the error processing and adjustment of internal motor representations (Miall \& Wolpert, 1996), would index the degree of cognitive control activation that influences plan-based control during motor sequence learning. Specifically, we predicted that sequence-specific learning performance that is representative of plan-based control, is related closely to the ERN and that changes in plan-based control would also reflect changes in ERN amplitudes. In addition, if short-term FAM training increased the degree of plan-based control in comparison to singlesession FAM, we predicted that the ERN amplitudes during sequence learning would also be further enhanced.

\section{Manipulation check: Cognitive task related spectral power in the alpha band $(8-13 \mathrm{~Hz})$}

Our motivation was to check that our experimental manipulation was successful in inducing different cognitive control states associated with the cognitive task that each group performed. Our previous experiments have relied on self-report measures and/or assumed that by performing the FAM practice, it would induce a different cognitive control state. Firstly, our results support that FAM practice in both a naïve (FAM1) and trained (FAM21) participants induced mental states that were quantitatively different from a general listening task performance. We found that alpha spectral power was higher across the central-midline, posterior-midline and posterior-right ROI for the Control group that performed LT, compared to FAM21 and FAM1 that performed FAM. Alpha band oscillatory activity is related to attentional processes (Klimesch, 2012) and provide an indication of whether attention is allocated internally or externally (Baldwin et al., 2017). Specifically, greater alpha power at parietal and occipital electrode locations have been found to be associated with failure to detect (Ergenoglu et al., 2004) or discriminate stimuli (Robertson, Manly, Andrade, Baddeley, \& Yiend, 1997). Prolonged periods of high alpha power, have also been found to be associated with lapses of attention to external stimuli (O'Connell et al., 2009). In addition, alpha power is expected to be increased during periods of mind wandering and activation of the default-mode network (DMN), relative to on task behaviour (Gouraud, Delorme, \& Berberian, 2017; Hinterberger, Schmidt, Kamei, \& Walach, 2014). Further, our results suggest that a single-session of FAM practice maybe able to induce relatively less distracted cognitive states and reduce mind-wandering during practice. Finally, our results are in line 
with other reported effects of meditation practice that showed a down regulation of alpha activity during successful meditation (Hinterberger et al., 2014), and supports that our experimental manipulation was successful.

\section{Single-session FAM and FAM training effects on motor sequence performance}

In comparison to control conditions, a single-session of FAM (FAM1) did not appear to show direct support for block-on-block RT improvements during sequence learning. FAM1 performed faster than Control in blocks 9 and 11, and Control was faster than FAM1 in blocks 6 and 7. The lack of differences in the rest of the blocks suggests that FAM1 and Control had equal performance. In the case of FAM1, it was during late-phase of sequence learning that block-on-block performance became as short as FAM21 and Control. In Control, the mid-phase of learning saw performance benefits against FAM21, but these benefits were not sustained into late-phase of learning. Chan et al. (2017) found performance benefits for block performances when a single-session of FAM was compared to a rest-only control condition. The current results between FAM1 and Control are more in agreement with our previous experiment (Chan et al., In review) that performing other cognitive tasks that actively engage cognitive control (such as CAT and LT) will not lead to performance deficits during sequence learning.

We found that short-term FAM training lead to faster performances during sequence learning compared to single-session FAM and a control condition. Specifically, FAM training led to the shortest block RT performances in majority of the sequence learning blocks compared to the other conditions. It should be emphasised that neither FAM1 nor Control had shorter block RTs compared to FAM21 during Sequence Learning. Firstly, this provides direct behavioural support that increased FAM training results in reinforcing single-session FAM practice benefits for general practice effects during sequence learning. Secondly, these general practice effects from FAM21 were evident from the early-phase of sequence learning (blocks 1-4) evidenced by shorter block RTs compared to FAM1 and Control. This pattern early-phase block-by-block performance advantages is suggestive that FAM21 had increased levels of top-down control right from the onset of sequence learning. This is consistent with the notion that increased levels of top-down control reinforced persistence towards the goal of speed and accuracy in processing stimuli information resulting in greater general practice effects (Hommel \& Colzato, 2017a). Greater general practice effects are also an indication of increased stimulus-based control to resolve the local competition between possible targets during trial presentation (Hommel \& Colzato, 2017a; Tubau, Hommel, et al., 2007). 
FAM21 maintained shorter block-on-block performances throughout sequence learning and it was only in the late-phase of sequence learning (blocks 8-12) that FAM1 exhibited similar RT performances to FAM21. Control only had similar performance in block 12. This maybe an indication that FAM1 and Control had increased levels of top-down control to support general practice effects only during the latter part of sequence learning. No differences in block RT between the last sequence learning block 12, transfer block 13 and final sequence learning 14 were evident between the groups. This indicates that sequencespecific learning effects and interference effects between the groups were equal and suggest that on a behavioural level, there were no differences in plan-based control (Chan et al., 2017).

The behavioural results are consistent with our prediction that increased FAM training will result in general learning performances during sequence learning, compared to the performance benefits already demonstrated in single-session FAM (Chan et al., 2017, In review; Immink et al., 2017). It appears to support that that effects seen in FAM21 were benefits for general practice instead of sequence-specific learning or interferences. This suggests that FAM21 increased stimulus-based control during learning to establish efficient ways for stimulus-response mapping (Abrahamse \& Noordzij, 2011; Tubau, Hommel, et al., 2007) that benefits accurate and rapid responses during sequence performance. Whilst the behavioural results support this interpretation, we also aimed to further understand the differences in neurophysiological correlates of sequence learning following single-session FAM practice and training.

\section{N200 and stimulus-based control: Effects from single-session FAM and FAM training}

No differences between at a broader group level for mean amplitude in the N200 component during Familiarisation was found, which was in line with the behavioural results. Although differences during Familiarisation showed that some of the different ROIs were interacting between the groups, no consistent pattern emerged. There are two major factors that limits our ability to interpret the N200 Familiarisation results meaningfully, first, that stimulus presentation in Familiarisation consisted of pseudo-random stimuli which served as a way for participants to orientate themselves to the SRTT. Structure of stimuli greatly affects cognitive control in sequence learning and hence adding session as a factor for comparison is essentially a flawed approach due to inequivalent stimuli across the sessions. Second, the number of blocks were also different and as such total trial numbers between Familiarisation and Sequence Learning were unequal. 
Twenty days of once daily FAM training (FAM21) further enhanced the N200 effects of single-session FAM. Specifically, our results clearly show the same pattern that across anterior and central ROIs, FAM21 had the greatest level of negativities for the N200 compared to FAM1 and Control. This pattern of activation across the ROIs for the N200 amplitude appears to follow a gradient that is moderated by the volume of FAM training: the least amount of negativity was exhibited by the Control; greater negativities were found in FAM1; and FAM21 exhibiting greatest levels of negativities for the N200 during Sequence Learning. First, performing a single-session of FAM (FAM1) in comparison to performing a LT (Control), significantly enhanced the negativity in the N200 across all anterior and central ROIs during Sequence Learning. The N200 activity in the anterior and central ROIs are highly suggestive of FPC and PFC contributions, which are key cortical structures involved with top-down control (Koechlin et al., 2003; Stuss \& Knight, 2013). Secondly, performing short-term FAM training further enhanced N200 amplitudes in comparison to single-session FAM across all anterior and central ROIs during Sequence Learning. The only exception was in the posterior-left ROI, where FAM21 had lower negativity compared to FAM1, but was still more negative than Control.

Our results of N200 were consistent with the work Moore et al. (2012) that investigated the effects of a FAM training in a longitudinal study design on the Stroop task. The authors found that posterior channels were associated with increased activation in left medial and lateral occipitotemporal areas and were contributors for an increase in N200 amplitudes following FAM training over a 16-week period. The authors attributed this increase to more efficient perceptual discrimination of stimuli because of increased focused attentional resources that were resultant of meditation training. In essence, perceptual discrimination is considered only one part of cognitive control operations that support the ability to discern between targets and non-targets when there are competing at a local level (Cahn \& Polich, 2006; Hommel et al., 2001; Polich, 2007). Which may be the reason why our posterior channels did not show interacting effects. Instead, our results of increased N200 amplitudes in the anterior and central ROIs are much more in line with the interpretation of contributions from the cortical-subcortical network connected to posterior medial frontal cortex and dorsomedial prefrontal cortex underlying increased top-down control (Danielmeier et al., 2011; Ullsperger, Fischer, et al., 2014). Essentially, these areas are activated to support increased top-down with the goal of maintaining sequence performance by processing targets swiftly and accurately (Ullsperger, Fischer, et al., 2014; Wessel, Klein, Ott, \& Ullsperger, 2014). To further discriminate if these cognitive control processes were linked to stimulus- 
1042

1043

1044

1045

1046

1047

1048

1049

1050

1051

1052

1053

1054

1055

1056

1057

1058

1059

1060

1061

1062

1063

1064

1065

1066

1067

1068

1069

1070

1071

1072

1073

1074

1075

based control in sequence learning, we had to establish links between sequence learning indices and N200 amplitudes. The results of our model-based approach found that general practice effects (Chan et al., 2017, In review), provided the best model fit as a predictor for N200 amplitudes compared to other sequence learning indices. This data-driven approach supported our prediction that N200 amplitude fluctuations can index the degree of stimulusbased control that is closely related to the behavioural measure of general practice effects.

The modelling presented an intriguing and obvious pattern across the anterior and central ROIs: the FAM21 group showed that initial levels of general practice effects are marked by increased N200 amplitudes, indicating greater levels of top-down control activation. However, as higher levels of general practice effects are achieved, N200 amplitudes were decreasing, and the rate of decline was much higher than that of FAM1 and Control. The Control group also showed a similar pattern when compared to FAM21 in the anterior and central ROIs but with less dramatic rate of decline in N200 amplitudes. The modelling also showed that at the highest levels of general practice effect improvements, Control group had higher relative N200 amplitudes compared to FAM21, which indicates greater top-down activation. The only exception for this was in the central-right ROI between FAM21 and Control. In contrast to FAM21, the FAM1 group did not show the same level of consistency in the anterior and central ROIs with very little change in N200 amplitudes from initial levels to higher levels of general practice effects. Opposed effects compared to FAM21 were even observed in the anterior (left and midline) ROIs, where increased N200 amplitudes were found to support higher levels of general practice effects. At the highest levels of general practice effect improvements, FAM1 group had higher amplitudes compared to FAM21, which also indicates greater top-down activation.

The key interpretation of the results is that short-term FAM training affords much greater levels of top down control during initial levels of general practice that supports much faster responding during motor sequence learning, in comparison to single-session FAM practice or LT control. However, FAM training will facilitate less top-down activation to support higher levels of general practice effects compared to single-session FAM and LT control. Earlier, we proposed that the N200 indexes the level of top-down activation and stimulus-based control required to perform sequence learning successfully. At first our modelling appears paradoxical considering that we showed that mean N200 amplitude were higher across sequence learning together, with much shorter block-on-block behavioural RT outcomes after FAM training. A very important point that must be considered in the SRTT paradigm is that learning is presumed to be based around block-on-block RT improvements. 
1076

1077

1078

1079

1080

1081

1082

1083

1084

1085

1086

1087

1088

1089

1090

1091

1092

1093

1094

1095

1096

1097

1098

1099

1100

1101

1102

1103

1104

1105

1106

1107

However, if enhanced performances in block RT are demonstrated initially, then throughout the course of sequence learning, less 'learning improvement' will be revealed. Enhanced initial performance would therefore appear to suppress general practice effects improvements, which is very misleading. Our modelling clarifies this by adding an additional layer of interpretation and shows that short-term FAM training not only increases top-down control activation, but that cognitive control is more dynamic in the sense that less activation is needed to support higher levels of general practice expertise (Daltrozzo \& Conway, 2014) compared to the other conditions. Reductions in amplitudes during high expertise performances are essentially evidences for the cognitive system achieving efficiency for better performances (Ansari \& Derakshan, 2011; Fan, McCandliss, Sommer, Raz, \& Posner, 2002).

Our modelling of the N200 supports that there is additional benefit from short-term FAM training in both stimulus-based control (Chan et al., 2017, In review; Immink et al., 2017) and more effective dynamic modulation of top-down control; increased top-down when needed to maintain performance and becoming more efficient at high levels of general practice effects of sequence learning performance.

\section{ERN and plan-based control: Effects from single-session FAM and FAM training}

Although the results of the ERN component during Familiarisation showed group and sagittality effects, there was no consistent pattern that emerged based on the three sagittal regions. On a broader level, mean amplitude of the ERN component across the groups and all ROIs were not different during Familiarisation.

The results of the ERN component during Sequence Learning also did not showcase any group and ROIs effects. However, the effects from the group and sagittality interaction showed that FAM1 had increased negativities for the ERN in the anterior, central and posterior ROI compared to the Control. FAM1 also had increased negativity for the ERN compared to FAM21 in the anterior and posterior ROI. FAM21 had increased negativity for the ERN compared to Control only in the anterior and central ROIs. Across all groups negativities for the ERN were greatest in the central region. The effects from the group and laterality interaction showed a very consistent pattern that appeared in a gradient manner. Across left, midline and right ROIs, FAM1 showed the greatest level of negativity for the ERN, with FAM21 lower and different from FAM1. FAM21 compared to Control had increased negativities for the ERN, and Control showed the least amount of negativity for the ERN across all laterality ROIs and groups. 
The results of our model-based approach and for the ERN did not support our hypothesis that sequence-specific learning would serve as a behavioural index of plan-based control, and thus be closely related to the ERN. Specifically, there was no support for sequence-specific learning to be the best model fit as a predictor for ERN amplitudes. In the ERN and SRTT study by Beaulieu et al. (2014), it was found that sequence-specific learning correlated better than general learning (indexed by overall RT improvement from first to last block) with ERN change (from early to late learning). Our models instead revealed that the general learning index (like the N200) provided the better model fit as a predictor for ERN amplitudes. However, the correlations in Beaulieu et al. (2014) was predicated on the neural activity of one single electrode channel $(\mathrm{Cz})$. This is limitation because the ERP methodology is known for its poor signal-to-noise ratio in individual electrodes (Luck et al., 2000) and therefore the usage of single channels is typically avoided. In our case we considered our approach of analysis to be an improvement over the work of Beaulieu et al. (2014). We created ROIs to pool channels and enhance our ability to detect signal from noise in the neural activity, whilst accounting for these ROIs factorially in our models. Our current approach showed that the anterior-midline and central-midline still exhibited the greatest levels of negativity for the ERN and therefore in agreement with observations that frontocentral areas are the significant contributors of the ERN component (Carter et al., 1998; Ullsperger, Danielmeier, \& Jocham, 2014; Ullsperger, Fischer, et al., 2014). In addition, instead of using correlations to test relationships, the use of statistical models afforded the data to guide the relationships of behavioural measures that could predict changes in ERN amplitudes. Therefore, we are confident in the results that revealed general learning was the best predictor, although general learning did not significantly interact with the ERN amplitudes during Sequence Learning.

Another prediction that short-term FAM training would further increased errorprocessing alongside increase ERN amplitudes and enhanced plan-based control, compared to single-session FAM, during sequence learning was also not supported. There are no previous studies that investigated meditation effects for ERN and sequence learning. Larson et al. (2013) found no differences for the ERNs elicited during errors in a Flanker task between naïve participants that performed FAM prior to, and controls. Although our results may appear to be in contrast that FAM1 resulted in increased ERN amplitudes across laterality ROIs compared to FAM21, the results from both studies are not directly comparable. In the case of Larson et al. (2013), amplitudes were averaged over four central channels, whilst our laterality regions each contained averaged amplitudes from between 18 to 20 channels. 
1142 Because we did not find an effect specifically in the central-midline region (closest ROIs in comparison), we are hesitant to conclude that practice in a single-session of FAM would indeed increase ERN amplitudes. Another ERN cross-sectional study of experienced meditators versus non-meditating age-matched controls and meditation study by Teper $\&$ Inzlicht (2013) only found differences in the ERN attributed to indirect effects from conflict management skills that extended towards processing errors. Several authors have suggested that effects in ERN on task performance from meditation may require an extended time spent practising (> one year) to manifest any meaningful changes which maybe a reason for the lack of meaningful findings in our current experiment (Larson et al., 2013; Malinowski, 2013; Tang et al., 2015; Teper \& Inzlicht, 2013).

\section{Methodological strengths and limitations}

1153 Participants in the current study were naïve and blinded to the experimental aims. This was to limit potential influences associated with the expectations of the benefits in meditation. Another strength that added rigour to the present experiment was the inclusion of an active control condition in the form of an LT. Through our manipulation check, we did we showed the FAM and LT were quantitatively different in the sense that increased alpha power in the posterior region for in LT resembled that of mind-wandering processes. In contrast, FAM was had down-regulation of alpha in the posterior ROI that was more reflective of deeper states of internalised attention. The period of sequence training was relatively short (one session of 14 blocks) and therefore the influence of meditation across several sessions of learning may be different (multi-days with different SOC sequences). The transfer block in our experiment was designed as random sequence presentation, in hindsight, a different SOC would have been a better design to maintain structural comparison between the old SOC in the last learning block and the transfer block.

With regards to FAM, it is unknown if top-down control effects require reinstatement prior to learning and/or that varying FAM duration may produce different effects. The insertion of FAM practice in-between blocks has also not been investigated, which may further strengthen top-down control between block performances. Finally, to further understand the contribution of meditation on sequence learning, other factors such as personal predispositions, meditation styles, duration of practice and temporal placement should be investigated. Because we've shown that attentional and cognitive control states can be can be biased, it would also be valuable to investigate the reversed effect of sequence learning for meditation. This would show that effects from various preceding task may also influence the 
1175 quality of meditation practice. These are all important directions that aim to further

1176 understand the mechanistic actions by which cognitive control states enhance motor learning

1177 performance.

1178 Summary: Cognitive control enhancement of FAM for sequential performance

1179 This was the first experiment to bridge key areas of cognitive control theory in sequence

1180 learning and investigate if neurophysiological correlates of cognitive control could be

1181 enhanced following single-session FAM, and further enhanced by short-term FAM training.

1182 Firstly, our results support and have replicated the single-session FAM can increase top-down

1183 activation for the N200 and modulate stimulus-based control that supports general practice

1184 performance in line with previous behavioural work (Chan et al., 2017; Chan, Immink, \&

1185 Lushington, In review.). Our results also supported our predictions that FAM training further

1186 enhanced single-session FAM effects with even greater levels of top-down activation for the

1187 N200, for greater stimulus-based control that supports even higher levels of general practice

1188 performances. When N200 amplitudes were modelled against different performance

1189 predictors, general practice performance provided the best fit. This is the first instance to

1190 show that the N200 in the SRTT is closely related to the concept of stimulus-based control

1191 (Tubau, Hommel, et al., 2007) and may be a promising way to investigate changes in top-

1192 down control during sequence learning in future work. Most interestingly, our results

1193 revealed that FAM21 had the greatest rate of decline in N200 amplitude from initial to higher

1194 levels of general practice effects improvements, indicating a dynamic modulation of top-down

1195 control during sequence learning. These effects were most evident over the anterior and

1196 central regions when compared to other conditions. The results from activation in these ROIs

1197 is consistent with the interpretation of increased top-down activation in related structures such

1198 as the FPC and PFC to facilitate and maintain performance (Ullsperger, Danielmeier, et al.,

1199 2014; Ullsperger, Fischer, et al., 2014).

1200 Although we also found some effects for the ERN, our modelling against behavioural

1201 performance indices did not support our predictions. We did not find the ERN was closely

1202 related to sequence-specific learning and hence not truly representative of plan-based control.

1203 We also did not find differences in sequence-specific learning between that groups which

1204 could indicate that further FAM training does not facilitate these improvements. This notion

1205 could be further tested by increasing training, since effects were only found in meditators with

1206 more than one year of practice (Teper \& Inzlicht, 2013). 
Meditation, Cognitive Control and Sequence Learning, 46

\section{Acknowledgements}

1208 We thank David Burgess for providing a recording of the yoga nidra meditation used in this

1209 experiment. We also express gratitude to the lab researchers and research assistants that

1210 helped to collect some of the data in this study: Alex Chatburn, Andrew Corcoran, Christina

1211 Lubinus, Erica Wilkinson, Schae Matthews and Matthew Seals. 


\section{$\underline{\text { References }}$}

1213

1214

1215

1216

1217

1218

1219

1220

1221

1222

1223

1224

1225

1226

1227

1228

1229

1230

1231

1232

1233

1234

1235

1236

1237

1238

1239

1240

1241

1242

1243

1244

1245

1246

1247

1248

1249

1250

1251

1252

1253

1254

1255

1256

1257

1258

1259

1260

Abrahamse, E. L., Jimenez, L., Verwey, W. B., \& Clegg, B. A. (2010). Representing serial action and perception. Psychon Bull Rev, 17(5), 603-623. doi:10.3758/PBR.17.5.603

Abrahamse, E. L., \& Noordzij, M. L. (2011). Designing training programs for perceptualmotor skills: Practical implications from the serial reaction time task. Revue Européenne de Psychologie Appliquée/European Review of Applied Psychology, 61(2), 65-76. doi:http://dx.doi.org/10.1016/j.erap.2010.12.001

Abrahamse, E. L., Ruitenberg, M. F., de Kleine, E., \& Verwey, W. B. (2013). Control of automated behavior: insights from the discrete sequence production task. Front Hum Neurosci, 7, 82. doi:10.3389/fnhum.2013.00082

Alexander, W. H., \& Brown, J. W. (2010). Computational Models of Performance Monitoring and Cognitive Control. Topics in Cognitive Science, 2(4), 658-677. doi:doi:10.1111/j.1756-8765.2010.01085.x

Amer, T., Campbell, K. L., \& Hasher, L. (2016). Cognitive Control As a Double-Edged Sword. Trends Cogn Sci, 20(12), 905-915. doi:10.1016/j.tics.2016.10.002

Amer, T., \& Hasher, L. (2014). Conceptual Processing of Distractors by Older but Not Younger Adults. Psychol Sci, 25(12), 2252-2258. doi:10.1177/0956797614555725

Ansari, T. L., \& Derakshan, N. (2011). The neural correlates of cognitive effort in anxiety: effects on processing efficiency. Biol Psychol, 86(3), 337-348. doi:10.1016/j.biopsycho.2010.12.013

Baldwin, C. L., Roberts, D. M., Barragan, D., Lee, J. D., Lerner, N., \& Higgins, J. S. (2017). Detecting and Quantifying Mind Wandering during Simulated Driving. Front Hum Neurosci, 11(406). doi:10.3389/fnhum.2017.00406

Baldwin, K. B., \& Kutas, M. (1997). An ERP analysis of implicit structured sequence learning. Psychophysiology, 34(1), 74-86.

Bates, D., Mächler, M., Bolker, B., \& Walker, S. (2015). Fitting Linear Mixed-Effects Models Using lme4. 2015, 67(1), 48. doi:10.18637/jss.v067.i01

Beaulieu, C., Bourassa, M. E., Brisson, B., Jolicoeur, P., \& De Beaumont, L. (2014). Electrophysiological correlates of motor sequence learning. BMC Neurosci, 15(1), 102. doi:10.1186/1471-2202-15-102

Boisgontier, M. P., \& Cheval, B. (2016). The anova to mixed model transition. Neurosci Biobehav Rev, 68, 1004-1005. doi:10.1016/j.neubiorev.2016.05.034

Botvinick, M. M., Braver, T. S., Barch, D. M., Carter, C. S., \& Cohen, J. D. (2001). Conflict monitoring and cognitive control. Psychol Rev, 108(3), 624-652.

Burnham, K. P., \& Anderson, D. R. (2003). Model Selection and Multimodel Inference: A Practical Information-Theoretic Approach: Springer New York.

Cahn, B. R., \& Polich, J. (2006). Meditation states and traits: EEG, ERP, and neuroimaging studies. Psychol Bull, 132(2), 180-211. doi:10.1037/0033-2909.132.2.180

Carter, C. S., Braver, T. S., Barch, D. M., Botvinick, M. M., Noll, D., \& Cohen, J. D. (1998). Anterior cingulate cortex, error detection, and the online monitoring of performance. Science, 280(5364), 747-749.

Chan, R. W., Immink, M. A., \& Lushington, K. (2017). The influence of focused-attention meditation states on the cognitive control of sequence learning. Conscious Cogn, 55, 11-25. doi:http://dx.doi.org/10.1016/j.concog.2017.07.004

Chan, R. W., Immink, M. A., \& Lushington, K. (In review). A comparison of instantaneous effects from single-session focused attention meditation and computerised attention task on cognitive control during sequence learning.

Chan, R. W., Immink, M. A., \& Lushington, K. (In review.). The influence of focusedattention meditation states on the cognitive control of sequence learning. 
Clegg, B. A., Digirolamo, G. J., \& Keele, S. W. (1998). Sequence learning. Trends Cogn Sci, 2(8), 275-281.

Colzato, L. S., Sellaro, R., Samara, I., Baas, M., \& Hommel, B. (2015). Meditation-induced states predict attentional control over time. Conscious Cogn, 37, 57-62. doi:10.1016/j.concog.2015.08.006

Colzato, L. S., Szapora, A., Lippelt, D., \& Hommel, B. (2017). Prior Meditation Practice Modulates Performance and Strategy Use in Convergent- and Divergent-Thinking Problems. Mindfulness (N Y), 8(1), 10-16. doi:10.1007/s12671-014-0352-9

Colzato, L. S., van der Wel, P., Sellaro, R., \& Hommel, B. (2016). A single bout of meditation biases cognitive control but not attentional focusing: Evidence from the global-local task. Conscious Cogn, 39, 1-7. doi:10.1016/j.concog.2015.11.003

Curran, T., \& Keele, S. W. (1993). Attentional and nonattentional forms of sequence learning. Journal of Experimental Psychology: Learning, Memory, and Cognition, 19(1), 189202. doi:10.1037/0278-7393.19.1.189

Daltrozzo, J., \& Conway, C. M. (2014). Neurocognitive mechanisms of statistical-sequential learning: what do event-related potentials tell us? Front Hum Neurosci, 8, 437. doi:10.3389/fnhum.2014.00437

Danielmeier, C., Eichele, T., Forstmann, B. U., Tittgemeyer, M., \& Ullsperger, M. (2011). Posterior medial frontal cortex activity predicts post-error adaptations in task-related visual and motor areas. J Neurosci, 31(5), 1780-1789. doi:10.1523/JNEUROSCI.4299-10.2011

Davidson, R. J., \& Kaszniak, A. W. (2015). Conceptual and methodological issues in research on mindfulness and meditation. Am Psychol, 70(7), 581-592. doi:10.1037/a0039512

Donchin, E., Ritter, W., \& McCallum, C. (1978). Cognitive psychophysiology: The endogenous components of the ERP. New York: Academic Press.

Du, Y., Prashad, S., Schoenbrun, I., \& Clark, J. E. (2016). Probabilistic Motor Sequence Yields Greater Offline and Less Online Learning than Fixed Sequence. Front Hum Neurosci, 10(87). doi:10.3389/fnhum.2016.00087

Eimer, M., Goschke, T., Schlaghecken, F., \& Sturmer, B. (1996). Explicit and implicit learning of event sequences: evidence from event-related brain potentials. $J$ Exp Psychol Learn Mem Cogn, 22(4), 970-987.

Ergenoglu, T., Demiralp, T., Bayraktaroglu, Z., Ergen, M., Beydagi, H., \& Uresin, Y. (2004). Alpha rhythm of the EEG modulates visual detection performance in humans. Brain Res Cogn Brain Res, 20(3), 376-383. doi:10.1016/j.cogbrainres.2004.03.009

Fan, J., McCandliss, B. D., Sommer, T., Raz, A., \& Posner, M. I. (2002). Testing the efficiency and independence of attentional networks. J Cogn Neurosci, 14(3), 340347. doi:10.1162/089892902317361886

Ferdinand, N. K., Runger, D., Frensch, P. A., \& Mecklinger, A. (2010). Event-related potential correlates of declarative and non-declarative sequence knowledge. Neuropsychologia, 48(9), 2665-2674. doi:10.1016/j.neuropsychologia.2010.05.013

Folstein, J. R., \& Van Petten, C. (2008). Influence of cognitive control and mismatch on the N2 component of the ERP: a review. Psychophysiology, 45(1), 152-170. doi:10.1111/j.1469-8986.2007.00602.x

Gallant, S. N. (2016). Mindfulness meditation practice and executive functioning: Breaking down the benefit. Conscious Cogn, 40, 116-130. doi:10.1016/j.concog.2016.01.005

Gehring, W. J., Goss, B., Coles, M. G. H., Meyer, D. E., \& Donchin, E. (1993). A Neural System for Error Detection and Compensation. Psychol Sci, 4(6), 385-390.

Gouraud, J., Delorme, A., \& Berberian, B. (2017). Autopilot, Mind Wandering, and the Out of the Loop Performance Problem. Front Neurosci, 11, 541. doi:10.3389/fnins.2017.00541 
Gramfort, A., Luessi, M., Larson, E., Engemann, D., Strohmeier, D., Brodbeck, C., . . Hämäläinen, M. (2013). MEG and EEG data analysis with MNE-Python. Front Neurosci, 7(267). doi:10.3389/fnins.2013.00267

Gratton, G., Cooper, P., Fabiani, M., Carter, C. S., \& Karayanidis, F. (2017). Dynamics of cognitive control: Theoretical bases, paradigms, and a view for the future. Psychophysiology. doi:10.1111/psyp.13016

Hasenkamp, W., \& Barsalou, L. W. (2012). Effects of meditation experience on functional connectivity of distributed brain networks. Front Hum Neurosci, 6, 38. doi:10.3389/fnhum.2012.00038

Hasenkamp, W., Wilson-Mendenhall, C. D., Duncan, E., \& Barsalou, L. W. (2012). Mind wandering and attention during focused meditation: a fine-grained temporal analysis of fluctuating cognitive states. Neuroimage, 59(1), 750-760. doi:10.1016/j.neuroimage.2011.07.008

Hasher, L., Quig, M. B., \& May, C. P. (1997). Inhibitory control over no-longer-relevant information: adult age differences. Mem Cognit, 25(3), 286-295.

Hinterberger, T., Schmidt, S., Kamei, T., \& Walach, H. (2014). Decreased electrophysiological activity represents the conscious state of emptiness in meditation. Front Psychol, 5, 99. doi:10.3389/fpsyg.2014.00099

Holroyd, C. B., \& Coles, M. G. H. (2002). The neural basis of human error processing: reinforcement learning, dopamine, and the error-related negativity. Psychol Rev, 109(4), 679-709. doi:10.1037/0033-295X.109.4.679

Hommel, B. (1996). The cognitive representation of action: automatic integration of perceived action effects. Psychol Res, 59(3), 176-186.

Hommel, B., \& Colzato, L. S. (2017). Meditation and Metacontrol. Journal of Cognitive Enhancement, 1(2), 115-121. doi:10.1007/s41465-017-0017-4

Hommel, B., Musseler, J., Aschersleben, G., \& Prinz, W. (2001). The Theory of Event Coding (TEC): a framework for perception and action planning. Behav Brain Sci, 24(5), 849-878; discussion 878-937.

Immink, M. A., Colzato, L. S., Stolte, M., \& Hommel, B. (2017). Sequence Learning Enhancement Following Single-Session Meditation Is Dependent on Metacontrol Mode and Experienced Effort. Journal of Cognitive Enhancement, 1(2), 127-140. doi:10.1007/s41465-017-0019-2

Jack, B. I., Christopher, D. W., Gregory, L. C., \& Emanuel, D. (1980). The Event-Related Brain Potential as an Index of Display-Monitoring Workload. Hum Factors, 22(2), 211-224. doi:10.1177/001872088002200210

Jha, A. P., Krompinger, J., \& Baime, M. J. (2007). Mindfulness training modifies subsystems of attention. Cogn Affect Behav Neurosci, 7(2), 109-119.

Klimesch, W. (2012). alpha-band oscillations, attention, and controlled access to stored information. Trends Cogn Sci, 16(12), 606-617. doi:10.1016/j.tics.2012.10.007

Krigolson, O. E., Pierce, L. J., Holroyd, C. B., \& Tanaka, J. W. (2009). Learning to become an expert: reinforcement learning and the acquisition of perceptual expertise. J Cogn Neurosci, 21(9), 1834-1841. doi:10.1162/jocn.2009.21128

Larson, M. J., Steffen, P. R., \& Primosch, M. (2013). The impact of a brief mindfulness meditation intervention on cognitive control and error-related performance monitoring. Front Hum Neurosci, 7, 308. doi:10.3389/fnhum.2013.00308

Lee, D. J., Kulubya, E., Goldin, P., Goodarzi, A., \& Girgis, F. (2018). Review of the Neural Oscillations Underlying Meditation. Front Neurosci, 12(178). doi:10.3389/fnins.2018.00178

Luck, S. J., Woodman, G. F., \& Vogel, E. K. (2000). Event-related potential studies of attention. Trends Cogn Sci, 4(11), 432-440. 
Lustig, C., Hasher, L., \& Tonev, S. T. (2006). Distraction as a determinant of processing speed. Psychon Bull Rev, 13(4), 619-625. doi:10.3758/bf03193972

Lutz, A., Jha, A. P., Dunne, J. D., \& Saron, C. D. (2015). Investigating the phenomenological matrix of mindfulness-related practices from a neurocognitive perspective. Am Psychol, 70(7), 632-658. doi:10.1037/a0039585

Malinowski, P. (2013). Neural mechanisms of attentional control in mindfulness meditation. Front Neurosci, 7, 8. doi:10.3389/fnins.2013.00008

McCallum, W. C. (1980). Some Sensory and Cognitive Aspects of ERPs: a Review. In H. H. Kornhubek \& L. Deecke (Eds.), Prog Brain Res (Vol. 54, pp. 261-278): Elsevier.

Miall, R. C., \& Wolpert, D. M. (1996). Forward Models for Physiological Motor Control. Neural Networks, 9(8), 1265-1279. doi:https://doi.org/10.1016/S0893-6080(96)00035$\underline{4}$

Miyake, A., Friedman, N. P., Emerson, M. J., Witzki, A. H., Howerter, A., \& Wager, T. D. (2000). The unity and diversity of executive functions and their contributions to complex "Frontal Lobe" tasks: a latent variable analysis. Cogn Psychol, 41(1), 49-100. doi:10.1006/cogp.1999.0734

Moore, A., Gruber, T., Derose, J., \& Malinowski, P. (2012). Regular, brief mindfulness meditation practice improves electrophysiological markers of attentional control. Front Hum Neurosci, 6, 18. doi:10.3389/fnhum.2012.00018

Müller, N. G., \& Knight, R. T. (2002). Age-related changes in fronto-parietal networks during spatial memory: an ERP study. Cognitive Brain Research, 13(2), 221-234. doi:https://doi.org/10.1016/S0926-6410(01)00119-7

Näätänen, R. (1990). The role of attention in auditory information processing as revealed by event-related potentials and other brain measures of cognitive function. Behavioral and Brain Sciences, 13(2), 201-233. doi:10.1017/S0140525X00078407

Nash, J. D., \& Newberg, A. (2013). Toward a unifying taxonomy and definition for meditation. Front Psychol, 4, 806. doi:10.3389/fpsyg.2013.00806

Nissen, M. J., \& Bullemer, P. (1987). Attentional requirements of learning: Evidence from performance measures. Cognitive Psychology, 19(1), 1-32. doi:http://dx.doi.org/10.1016/0010-0285(87)90002-8

O'Connell, R. G., Dockree, P. M., Robertson, I. H., Bellgrove, M. A., Foxe, J. J., \& Kelly, S. P. (2009). Uncovering the neural signature of lapsing attention: electrophysiological signals predict errors up to $20 \mathrm{~s}$ before they occur. J Neurosci, 29(26), 8604-8611. doi:10.1523/JNEUROSCI.5967-08.2009

Oberauer, K., \& Kliegl, R. (2006). A formal model of capacity limits in working memory. Journal of Memory and Language, 55(4), 601-626. doi:https://doi.org/10.1016/j.jml.2006.08.009

Patel, S. H., \& Azzam, P. N. (2005). Characterization of N200 and P300: selected studies of the Event-Related Potential. Int J Med Sci, 2(4), 147-154.

Polich, J. (2007). Updating P300: an integrative theory of P3a and P3b. Clin Neurophysiol, 118(10), 2128-2148. doi:10.1016/j.clinph.2007.04.019

Richardson, M. J., Riley, M. A., \& Shockley, K. (2013). Progress in Motor Control: Neural, Computational and Dynamic Approaches: Springer New York.

Ritter, W., Simson, R., \& Vaughan, H. G., Jr. (1983). Event-related potential correlates of two stages of information processing in physical and semantic discrimination tasks. Psychophysiology, 20(2), 168-179.

Robertson, E. M. (2007). The serial reaction time task: implicit motor skill learning? $J$ Neurosci, 27(38), 10073-10075. doi:10.1523/JNEUROSCI.2747-07.2007 
Robertson, I. H., Manly, T., Andrade, J., Baddeley, B. T., \& Yiend, J. (1997). 'Oops!': performance correlates of everyday attentional failures in traumatic brain injured and normal subjects. Neuropsychologia, 35(6), 747-758.

Rodriguez-Fornells, A., Kurzbuch, A. R., \& Munte, T. F. (2002). Time course of error detection and correction in humans: neurophysiological evidence. J Neurosci, 22(22), 9990-9996.

Russeler, J., Kuhlicke, D., \& Munte, T. F. (2003). Human error monitoring during implicit and explicit learning of a sensorimotor sequence. Neurosci Res, 47(2), 233-240.

Rüsseler, J., Münte, T. F., \& Wiswede, D. (2018). On the influence of informational content and key-response effect mapping on implicit learning and error monitoring in the serial reaction time (SRT) task. Exp Brain Res, 236(1), 259-273. doi:10.1007/s00221017-5124-Z

Russeler, J., \& Rosler, F. (2000). Implicit and explicit learning of event sequences: evidence for distinct coding of perceptual and motor representations. Acta Psychol (Amst), 104(1), 45-67.

Saraswati, S. (2002). Yoga Nidra: Yoga Publications Trust.

Satterfield, J. H., Schell, A. M., Nicholas, T. W., Satterfield, B. T., \& Freese, T. E. (1990). Ontogeny of selective attention effects on event-related potentials in attention-deficit hyperactivity disorder and normal boys. Biol Psychiatry, 28(10), 879-903.

Saunders, B., Rodrigo, A. H., \& Inzlicht, M. (2016). Mindful awareness of feelings increases neural performance monitoring. Cognitive, Affective, \& Behavioral Neuroscience, 16(1), 93-105. doi:10.3758/s13415-015-0375-2

Schwab, J. F., Schuler, K. D., Stillman, C. M., Newport, E. L., Howard, J. H., \& Howard, D. V. (2016). Aging and the statistical learning of grammatical form classes. Psychol Aging, 31(5), 481-487. doi:10.1037/pag0000110

Slagter, H. A., Davidson, R. J., \& Lutz, A. (2011). Mental training as a tool in the neuroscientific study of brain and cognitive plasticity. Front Hum Neurosci, 5, 17. doi:10.3389/fnhum.2011.00017

Szczepanski, Sara M., \& Knight, Robert T. Insights into Human Behavior from Lesions to the Prefrontal Cortex. Neuron, 83(5), 1002-1018. doi:10.1016/j.neuron.2014.08.011

Tang, Y. Y., Holzel, B. K., \& Posner, M. I. (2015). The neuroscience of mindfulness meditation. Nat Rev Neurosci, 16(4), 213-225. doi:10.1038/nrn3916

Teper, R., \& Inzlicht, M. (2013). Meditation, mindfulness and executive control: the importance of emotional acceptance and brain-based performance monitoring. Soc Cogn Affect Neurosci, 8(1), 85-92. doi:10.1093/scan/nss045

Tubau, E., Hommel, B., \& Lopez-Moliner, J. (2007). Modes of executive control in sequence learning: from stimulus-based to plan-based control. J Exp Psychol Gen, 136(1), 4363. doi:10.1037/0096-3445.136.1.43

Ullsperger, M., Danielmeier, C., \& Jocham, G. (2014). Neurophysiology of performance monitoring and adaptive behavior. Physiol Rev, 94(1), 35-79. doi:10.1152/physrev.00041.2012

Ullsperger, M., Fischer, A. G., Nigbur, R., \& Endrass, T. (2014). Neural mechanisms and temporal dynamics of performance monitoring. Trends Cogn Sci, 18(5), 259-267. doi:10.1016/j.tics.2014.02.009

van Leeuwen, S., Singer, W., \& Melloni, L. (2012). Meditation increases the depth of information processing and improves the allocation of attention in space. Front Hum Neurosci, 6, 133. doi:10.3389/fnhum.2012.00133

Verstynen, T., Phillips, J., Braun, E., Workman, B., Schunn, C., \& Schneider, W. (2012). Dynamic Sensorimotor Planning during Long-Term Sequence Learning: The Role of 
Variability, Response Chunking and Planning Errors. PLoS One, 7(10), e47336. doi:10.1371/journal.pone.0047336

Verwey, W. B., Shea, C. H., \& Wright, D. L. (2015). A cognitive framework for explaining serial processing and sequence execution strategies. Psychon Bull Rev, 22(1), 54-77. doi:10.3758/s13423-014-0773-4

Verwey, W. B., \& Wright, D. L. (2014). Learning a keying sequence you never executed: evidence for independent associative and motor chunk learning. Acta Psychol (Amst), 151, 24-31. doi:10.1016/j.actpsy.2014.05.017

Wickens, C. D., Hutchins, S., Carolan, T., \& Cumming, J. (2013). Effectiveness of part-task training and increasing-difficulty training strategies: a meta-analysis approach. Hum Factors, 55(2), 461-470. 